\title{
KAT-7 Science Verification: Using Hi Observations of NGC 3109 to Understand its Kinematics and Mass Distribution
}

\author{
C. Carignan ${ }^{1}$, B. S. Frank, K. M. Hess, D. M. Lucero and T. H. Randriamampandry \\ Department of Astronomy, University of Cape Town, Private Bag X3, Rondebosch 7701, South Africa \\ and \\ S. Goedhart and S. S. Passmoor \\ SKA South Africa, The Park, Park Road, Pinelands, 7405, South Africa \\ ccarignan@ast.uct.ac.za
}

\begin{abstract}
Hi observations of the Magellanic-type spiral NGC 3109, obtained with the seven dish Karoo Array Telescope (KAT-7), are used to analyze its mass distribution. Our results are compared to what is obtained using VLA data. KAT-7 is the precursor of the SKA pathfinder MeerKAT, which is under construction. The short baselines and low system temperature of the telescope make it sensitive to large scale low surface brightness emission. The new observations with KAT7 allow the measurement of the rotation curve of NGC 3109 out to $32^{\prime}$, doubling the angular extent of existing measurements. A total Hi mass of $4.6 \times 10^{8} \mathrm{M}_{\odot}$ is derived, $40 \%$ more than what was detected by the VLA observations.

The observationally motivated pseudo-isothermal dark matter (DM) halo model can reproduce very well the observed rotation curve but the cosmologically motivated NFW DM model gives a much poorer fit to the data. While having a more accurate gas distribution has reduced the discrepancy between the observed RC and the MOdified Newtonian Dynamics (MOND) models, this is done at the expense of having to use unrealistic mass-to-light ratios for the stellar disk and/or very large values for the MOND universal constant $a_{0}$. Different distances or HI contents cannot reconcile MOND with the observed kinematics, in view of the small errors on those two quantities. As for many slowly rotating gas-rich galaxies studied recently, the present result for NGC 3109 continues to pose a serious challenge to the MOND theory.
\end{abstract}

Subject headings: techniques: interferometric - galaxies: individual: NGC 3109 - galaxies: kinematics and dynamics - galaxies: haloes - cosmology: dark matter

\section{Introduction}

On 2012 May 25, South Africa was awarded the construction of the mid-frequency array of the Square Kilometer Array (SKA), while Australia will build the low-frequency array. The SKA will consist of an ensemble of $3000 \sim 15 \mathrm{~m}$ dishes of which $80 \%$ will constitute the core portion in the Karoo desert while the remaining antennae will

\footnotetext{
${ }^{1}$ South African SKA Research Chair in MultiWavelength Astronomy
}

extend all the way to the 8 African partner countries, namely Botswana, Ghana, Kenya, Madagascar, Mauritius, Mozambique, Namibia and Zambia. It is expected that the full SKA will be completed around 2025. A precursor array of 64 dishes, MeerKAT, is already under construction by South Africa and should be ready for science operation in 2016. In preparation for these two large projects, a pre-precursor array comprising 7 dishes, KAT-7, was completed in December 2010. While its main purpose is to test technical solu- 
tions for MeerKAT and the SKA, scientific targets such as NGC 3109 were also observed during commissioning to test the Hi spectral line mode. In this paper, we compare over 100 hours of observations taken with $\mathrm{KAT}-7$ to previously obtained VLA data and perform a thorough analysis of the mass distribution of NGC 3109.

NGC 3109 is an SB(s)m galaxy (de Vaucouleurs et al. 1991) on the outskirts of the Local Group (van den Bergh 1994). It is even believed by certain authors to belong to the Local Group (e.g. Mateo 1998). While NGC 3109 looks like an Irregular galaxy on short exposures (Sandage 1961), it is clearly a spiral on longer exposures (Carignan 1985). Spiral arms are clearly visible, especially on the east side. This small spiral (scale length $\alpha^{-1}=1.2 \mathrm{kpc}$ ) is a Low Surface Brightness (LSB) system with $B(0)_{c}=$ 23.17 Carignan 1985). Its optical parameters are summarized in Table 1. One important parameter for mass modeling is the distance. Fortunately, because of its proximity, numerous Cepheids were observed in this Magellanic-type spiral, The most recent measurements are summarized in Table 2 , For this study, we adopt a distance of $1.30 \pm 0.02$ Mpc (Soszyński et al. 2006).

NGC 3109 is of significant scientific interest for two main reasons. Firstly, Jobin \& Carignan (1990) used observations with the hybrid VLA DnC configuration (synthesized beam of $36^{\prime \prime} \times$ $27^{\prime \prime}$ and velocity resolution of $10.3 \mathrm{~km} \mathrm{~s}^{-1}$ ) to perform a dynamical study of this galaxy, comparing the rotation curve (RC) derived from a tilted-ring analysis (see Sec. 3.4.1) to models composed of a luminous disk (stars \& gas) and of a dark isothermal (ISO) halo (see Sec. 4.1.1). Such a mass model provides an excellent fit to this nearly solid-body type RC. Combining the $\mathrm{HI} \mathrm{RC}$ with higher resolution $\mathrm{H} \alpha$ kinematical data, Blais-Ouellette, Amram \& Carignan (2001) also obtained a very good ISO Dark Matter (DM) model but a much less accurate fit for the cosmologically motivated NFW (Navarro, Frenk \& White 1997) DM model.

Recently, it was also shown that a MOdified Newtonian Dynamics (Milgrom 1983, 1988) model (MOND) could not reproduce the NGC 3109's RC (Randriamampandry 2013), at least with the data available. With our new data, it should be possible to compare DM (ISO and NFW) models to MOND (no dark matter) models and see
Table 1: Optical parameters of NGC 3109 (DDO 236).

\begin{tabular}{lcc}
\hline \hline Parameter & & Ref \\
\hline Morphological type & $\mathrm{SB}(\mathrm{s}) \mathrm{m}$ & $(1)$ \\
Right Ascension (J2000) & $10^{\mathrm{h}} 03^{\mathrm{m}} 06.7^{\mathrm{s}}$ & $(1)$ \\
Declination (J2000) & $-26^{\mathrm{o}} 09^{\prime} 32^{\prime \prime}$ & $(1)$ \\
Distance Modulus $(m-M)_{0}$ & $25.57 \pm 0.02$ & $(2)$ \\
Distance (Mpc) & $1.30 \pm 0.02$ & $(2)$ \\
Scale (pc arcmin $\left.{ }^{-1}\right)$ & 378 & \\
Isophotal major diameter, $D_{25}$ & $14.4^{\prime}$ & $(3)$ \\
Holmberg radius, $R_{H O}$ & $13.3^{\prime}$ & $(3)$ \\
Total apparent $B$ magnitude & 10.27 & $(4)$ \\
Corrected apparent $B$ mag. & 9.31 & $(1)$ \\
Absolute $B$ magnitude & -16.26 & $(2)$ \\
Exponential disk parameters: & & \\
Central $B(0)_{c}$ & 23.17 & $(4)$ \\
Scale length, $\alpha^{-1}(\mathrm{kpc})$ & 1.2 & $(4)$ \\
\hline
\end{tabular}

References. - (1) de Vaucouleurs et al. (1991); (2) Soszyński et al. (2006); (3) Jobin \& Carignan (1990); (4) Carignan (1985).

Table 2: Cepheids distance estimates for NGC 3109 .

\begin{tabular}{lc}
\hline \hline Reference & (Mpc) \\
\hline Soszvński et al. (2006) & $1.30 \pm 0.02$ \\
Pietrzvński et al. (2006) & $1.28 \pm 0.03$ \\
Musella. Piotto \&Capaccioli (1997) & $1.36 \pm 0.10$ \\
Capaccioli, Piotto \& Bresolin (1992) & $1.26 \pm 0.10$ \\
Mean Cepheids distance & $<1.30 \pm 0.04>$ \\
\hline
\end{tabular}

if NGC 3109 really challenges the MOND theory. This is not the first time that NGC 3109 poses problems to MOND (see e.g. Sanders 1986; Begeman. Broeils \& Sanders 1991).

Secondly, Barnes \& de Blok (2001) used $21 \mathrm{~cm}$ Multibeam data with the Parkes $64 \mathrm{~m}$ dish (beam $\sim 15.5^{\prime}$ and velocity resolution of $1.1 \mathrm{~km} \mathrm{~s}^{-1}$ ) to study the environment of NGC 3109. They provide a compelling argument that the warp in the Hi disk of NGC 3109 could be due to a dynamical encounter with the Antlia dwarf. This is also suggested by the elongation of the optical isophotes 
of NGC 3109 toward the south (Jobin \& Carignan 1990) and those of Antlia in the direction of NGC 3109 (Penny et al. 2012).

However, recent derivation of various merger and/or interaction parameters (e.g. asymmetry, clumpiness) by Pimbblet \& Couch (2012) are consistent with Antlia being an undisturbed dwarf elliptical. In fact, despite its dSph appearance, Antlia is better classified as a dSph/dIrr transition type (Grebel. Gallagher \& Harbeck 2003) because of its high HI content. With high sensitivity and mainly better spatial resolution observations (KAT-7 vs HIPASS), it should be possible to map much better the traces of that interaction, if it exists. An encounter/interaction that has significantly altered NGC3109's kinematics would give less weight to the finding that MOND cannot reproduce the rotation curve.

The remainder of this paper is as follows. In Sec. 2, a description of the new radio interferometer KAT-7 is given. Sec. 3.1 describes in details the new Hi data obtained with KAT-7, Sec. 3.2 those from the VLA-ANGST survey and Sec. 3.3 compares the different data sets. Sec. 3.4 derives the optimal RC that is used for the DM (ISO and NFW) and MOND models of Sec. 4. A discussion follows in Sec. 5 and a summary of the results and the final conclusions are given in Sec. 6.

\section{A New Radio Interferometer: KAT-7}

The seven-dish KAT-7 array, shown in Fig. 1 was built as an engineering testbed for the 64dish Karoo Array Telescope, known as MeerKAT, which is the South African pathfinder for the Square Kilometer Array (SKA). KAT-7 and MeerKAT are located close to the South African SKA core site in the Northern Cape's Karoo desert region. KAT -7 is remotely controlled from Cape Town, some $800 \mathrm{~km}$ away from the site. Construction of the array started in early 2008 and was completed in December 2010, with "first light" fringes obtained between two antennas in December 2009. The instrument is now in its science verification stage.

The array is extremely compact, with baselines ranging between $26 \mathrm{~m}$ to $185 \mathrm{~m}$. The KAT-7 layout was determined using the optimization algorithm described in de Villiers (2007), which determined a layout with a Gaussian UV

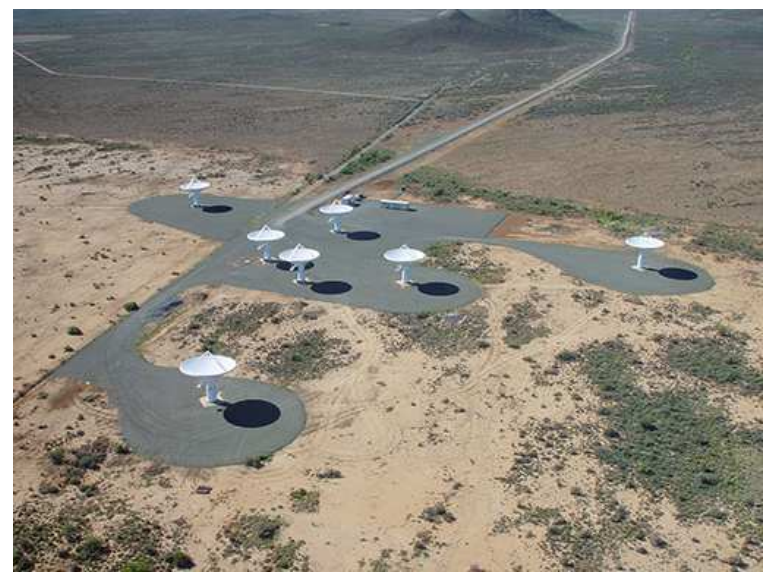

Fig. 1.- Aerial view of the KAT -7 array in the Northern Cape's Karoo desert, South Africa.

distribution for a specified observation setting. The observation setting being optimized in this case was an 8 hour track (symmetric hour angle range), on a target at a -60 degree declination. The optimization objective was a Gaussian UV distribution at $1.4 \mathrm{GHz}$, yielding a Gaussian synthesized beam with low sidelobes. Several randomly seeded layouts were generated and were evaluated for a set of observation options (time durations: snapshot, $4 \mathrm{hr}, 8 \mathrm{hr}, 12 \mathrm{hr}$; declinations: $0,-30,-60,-90$ degrees). The layout selected had the lowest sidelobes for the largest number of test observation settings considered. The antenna layout can be found at https://sites.google.com/a/ska.ac.za/public/kat7.

The KAT-7 dishes have a prime-focus alt-az design with a F/D of 0.38 , optimized for single-pixel L-band feeds. The low noise amplifiers (LNAs) for the feeds are cryogenically cooled to $80 \mathrm{~K}$ using Stirling coolers. The key system specifications for KAT-7 are summarized in Table 3. The digital backend of the system is an FPGA (Field Programmed Gate Array)-based, flexible packetised correlator using the Reconfigurable Open Architecture Computing Hardware (ROACH: https://casper.berkeley.edu/wiki/ROACH), which is a flexible and scalable system enabling spectral line modes covering a wide range of resolutions. Table 4 gives the details of the recently commissioned correlator modes. Digital filters give a flat bandpass over the inner $75 \%$ of the band with a 
rapid roll-off at the edges of the band.

Table 3: KAT-7 specifications.

\begin{tabular}{lc}
\hline \hline Parameter & Value \\
\hline Number of antennas & 7 \\
Dish diameter & $12 \mathrm{~m}$ \\
Min baseline & $26 \mathrm{~m}$ \\
Max baseline & $185 \mathrm{~m}$ \\
Frequency range & $1200-1950 \mathrm{MHz}$ \\
Max instantaneous bandwidth & $256 \mathrm{MHz}$ \\
Polarisation & Linear H \& V \\
$\mathrm{T}_{\text {sys }}$ & $26 \mathrm{~K}$ \\
Aperture efficiency & 0.65 \\
System Equivalent Flux Density & $1000 \mathrm{Jy}$ \\
Latitude & $-30: 43: 17.34$ \\
Longitude & $21: 24: 38.46$ \\
Elevation & $1038 \mathrm{~m}$ \\
Digital back-end & ROACH boards \\
\hline
\end{tabular}

Table 4: KAT-7 correlator modes.

\begin{tabular}{lccc}
\hline \hline mode & $\begin{array}{c}\text { total BW } \\
(\mathrm{MHz})\end{array}$ & $\begin{array}{c}\text { number of } \\
\text { channels }\end{array}$ & $\begin{array}{c}\text { channel BW } \\
(\mathrm{kHz})\end{array}$ \\
\hline c16n2M4k & 1.5625 & 4096 & 0.381 \\
c16n7M4k & 6.25 & 4096 & 1.526 \\
c16n25M4k & 25 & 4096 & 6.104 \\
c16n400M4k & 256 & 1024 & 390.625 \\
\hline
\end{tabular}

CASA (Common Astronomy Software Applications; McMullin et al. 2007) is the standard data reduction package being used for the reduction of the $\mathrm{KAT}-7$ data and is anticipated to be used for MeerKAT.

\section{HI Observations of NGC 3109}

The KAT-7 Hi observations of NGC 3109 provide a unique opportunity to simultaneously achieve Hi spectral-line science verification and an original scientific result. They complement the high spatial resolution $\left(\sim 10^{\prime \prime}\right)$ but small field of view $\left(\sim 30^{\prime}\right)$ of the VLA-ANGST data (Ott et al. 2012) and the high sensitivity $\left(\sim 10^{17} \mathrm{~cm}^{-2}\right)$ but low spatial resolution $\left(\sim 15.5^{\prime}\right)$ Multibeam data (Barnes \& de Blok 2001). With its short baselines and low system temperature $\left(\mathrm{T}_{\text {sys }} \sim 26 \mathrm{~K}\right), \mathrm{KAT}-$
7 is very sensitive to low surface brightness and large scale Hi emission, characteristic of the signal expected from NGC 3109.

\subsection{KAT -7 data on NGC 3109}

In order to observe the HI in both NGC 3109 and Antlia, plus possible signs of interaction between the two, a mosaic of 3 fields was obtained to have good sensitivity over a region of $1.5^{\circ}(\mathrm{EW}) \times$ $3^{\circ}$ (NS). The data was collected over 13 observing sessions between 2012 November 20 and 2012 December 26 using the c16n7M4k spectral line mode (Table 4) for a median of 11 hours in each session and a total of $122^{\mathrm{h}} 43^{\mathrm{m}} 56^{\mathrm{s}}$, including calibration. This yielded a total time on source of $\sim 25$ hours for each pointing.

The first three sessions were taken with 6 cold antennae, but the entire array was available for the remaining 10 observing sessions. The roughly 1 degree beam of KAT-7 is just large enough to image NGC 3109 in a single pointing. We used three pointings positioned in a straight line and extending slightly to the SE to mosaic the region between NGC 3109 and Antlia. The distance between pointings was chosen to give a uniform coverage between the phase centers. The c16n7M4k correlator mode gives velocity channels of $0.32 \mathrm{~km}$ $\mathrm{s}^{-1}$ over a flat bandpass of $\sim 1000 \mathrm{~km} \mathrm{~s}^{-1}$, centered at $1417 \mathrm{MHz}$. The large bandwidth allows to collect Hi data on background galaxies in the field.

The basic data reduction was done in CASA 3.4.0 and 4.0.0. More advanced analysis was done using either AIPS (Greisen 2003), MIRIAD (Sault, Teuben \& Wright 1995) and/or GIPSY (van der Hulst et al. 1992). To start with, the data was flagged in an automated way to discard data for shadowing and flux calibrators below 20 degrees in elevation. The data was additionally examined as a function of frequency and baseline, and flagged by hand.

This testing of the HI spectral line mode led to the discovery of faint, very narrow, internally generated radio frequency interference (RFI) originating along the signal path, which has since been successfully eliminated in KAT-7 by the insertion of a low-pass filter. The RFI in our data is antenna dependent and only affects about 30 channels out of the central 3000 on three antennae. One of the 
primary goals of the science verification phase is exactly to identify these type of problems and correct for them.

The standard interferometric data reduction strategy that has been employed for decades in AIPS and Miriad has been used. Each of the 13 observing sessions was reduced individually. Continuum subtraction was accomplished by selecting line free channels and using a first order fit. KAT7 does not use Doppler tracking, and CASA does not fully recognize frequency keywords, so special care was taken to produce image cubes with the proper velocity coordinates. This was a three steps process accomplished by:

- setting the MEAS_FREQ_REF and REF_ FREQUENCY keywords in the SPECTRAL WINDOW table,

- specifying the reference frequency and setting the output frame to optical, barycentric in CVEL, and

- specifying the rest frequency again in the task CLEAN.

The calibration was applied and the three mosaic pointings were then SPLIT from the calibration sources. The data were averaged in time from 5 to 10 second integrations, and spectrally from $0.32 \mathrm{~km} \mathrm{~s}^{-1}$ to $1.28 \mathrm{~km} \mathrm{~s}^{-1}$ channels. All 13 data sets were then combined in CONCAT.

The data was imaged using the mosaic mode and the multi-scale clean option. Three cubes were produced by applying natural (na), uniform (un), and neutral (ro: robust=0) weighting (Table 5) to the uv data. The robust $=0$ cube was cleaned interactively using a mask to select regions of galaxy emission by hand in each channel. After each major clean cycle, the mask was expanded to include regions of fainter galaxy emission. After a final mask was created, the cube was reproduced using the final mask in a non-interactive clean down to the noise threshold. All cubes and images were produced using the same mask derived from the robust $=0$ weighted cube. This provided a compromise between surface brightness sensitivity to large-scale emission and a low noise threshold, while mitigating confusion from sidelobes and low-level artifacts due to instrument calibration.

In addition to NGC 3109, Antlia, ESO 499G037 and ESO 499-G038, an HI cloud which has
Table 5: Parameters of the KAT-7 observations.

\begin{tabular}{lr}
\hline \hline Parameter & Value \\
\hline Start of observations & 20 nov. 2012 \\
End of observations & 26 dec. 2012 \\
Total integration per pointing & 24.75 hours \\
FWHM of primary beam & $58.67^{\prime}$ \\
Total Bandwidth & $6.25 \mathrm{MHz}$ \\
Central frequency & $1417 \mathrm{MHz}$ \\
Channel Bandwidth $(4 \times 1.526 \mathrm{kHz})$ & $6.1 \mathrm{kHz}$ \\
Number of channels $(4096 / 4)$ & 1024 \\
Channel width $\left(4 \times 0.32 \mathrm{~km} \mathrm{~s}{ }^{-1}\right)$ & $1.28 \mathrm{~km} \mathrm{~s}{ }^{\prime \prime}$ \\
Maps gridding & $55^{\prime \prime} \times 55^{\prime \prime}$ \\
Maps size & $128 \times 256$ \\
Flux/bandpass calibrator & $3 \mathrm{C} 138$ \\
Phase calibrator & $0118-317$ \\
\hline Robust = 0 weighting function & $212^{\prime \prime} \times 201^{\prime \prime}$ \\
FWHM of synthesized beam & $0.6 \mathrm{mJy} / \mathrm{beam}$ \\
RMS noise & 0.014 \\
Conversion ${ }^{\circ} \mathrm{K} /(1$ mJy/beam $)$ & $264^{\prime \prime} \times 233^{\prime \prime}$ \\
\hline Natural weighting function & $3.7 \mathrm{mJy} /$ beam \\
FWHM of synthesized beam & 0.010 \\
RMS noise (mJy/beam) & $203^{\prime \prime} \times 196^{\prime \prime}$ \\
Conversion ${ }^{\circ} \mathrm{K} /(1$ mJy/beam $)$ & $9.1 \mathrm{mJy} /$ beam \\
\hline Uniform weighting function & 0.015 \\
FWHM of synthesized beam & \\
RMS noise & \\
Conversion ${ }^{\circ} \mathrm{K} /(1$ mJy/beam $)$ & \\
\hline
\end{tabular}

no known optical counterpart was serendipitously discovered to the north of ESO 499-G038 at a similar velocity (Figure 2). The channels that contain this emission are remarkably clean and uniform in their noise characteristics. By contrast the channels which contain the brightest $\mathrm{HI}$ emission from NGC 3109 contain artifacts from sidelobes of the telescope's synthesized beam, which we have been unable to remove completely. The noise value in these channels is three times higher than elsewhere in the cube. For our analysis of NGC 3109, the cube produced with na weighting is used, except for the map showing the sum of all the channels (Figure 2) which used the ro weighting scheme. The parameters of the KAT-7 observations are summarized in Table 5 .

Fig. 2 shows the total intensity map for all the channels of the data cube. The lowest contour is at $1.0 \times 10^{19}$ atoms $\mathrm{cm}^{-2}$. Besides NGC 
3109 to the north and Antlia to the south, the two background galaxies ESO 499-G037 (10:03:42 $-27: 01: 40 ; \mathrm{V}_{\text {sys }}=953 \mathrm{~km} \mathrm{~s}^{-1}$ ) and ESO 499-G038 $\left(10: 03: 50-26: 36: 46 ; \mathrm{V}_{\text {sys }}=871 \mathrm{~km} \mathrm{~s}^{-1}\right)$ are clearly visible. More details about these two systems will be given in the Appendix. As mentioned earlier, the small cloud between ESO 499-G038 and NGC 3109 has no obvious optical counterpart. However, it is clearly associated with ESO 499-G038 and not NGC 3109 , being at a velocity $>900 \mathrm{~km}$ $\mathrm{s}^{-1}$.

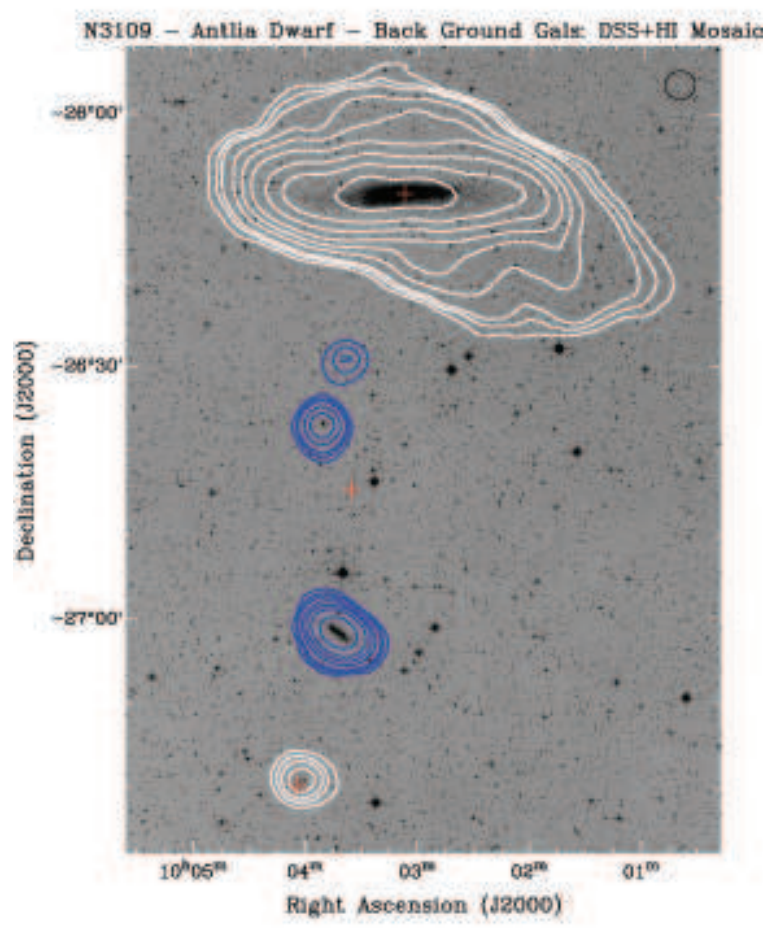

Fig. 2.- Sum of the channel maps of the KAT-7 Hi mosaic. The centers of the 3 fields are shown with red crosses. The contours are 0.1, 0.2, 0.3, $0.6,1.0,1.6,3.2,6.4,12.8 \times 10^{20}$ atoms $\mathrm{cm}^{-2}$, superposed on a DSS B image. NGC $3109\left(V_{\text {sys }}=\right.$ $\left.404 \mathrm{~km} \mathrm{~s}^{-1}\right)$ at the top and Antlia $\left(V_{\text {sys }}=360 \mathrm{~km}\right.$ $\left.\mathrm{s}^{-1}\right)$ at the bottom are shown with white contours. The two background galaxies ESO 499-G037 ( $V_{\text {sys }}$ $\left.=953 \mathrm{~km} \mathrm{~s}^{-1}\right)$, ESO $499-\mathrm{G} 038\left(V_{\text {sys }}=871 \mathrm{~km}\right.$ $\left.\mathrm{s}^{-1}\right)$ and its associated Hi cloud $\left(V_{s y s}=912 \mathrm{~km}\right.$ $\mathrm{s}^{-1}$ ) are shown with blue contours. The synthesized beam is shown in the upper-right corner.

The natural weighted cube is the best place to look for low-surface brightness emission between NGC 3109 and Antlia, but there is no obvious evidence of it there. In fact the elevated noise and strong sidelobes in the channels, which contain bright NGC 3109 data, prevent us from detecting lower surface brightness emission and limit what we can learn from smoothing the data. This work on NGC 3109 showed that better models of the KAT-7 primary beam are needed for calibration before we can go deeper.

Fig. 3 shows the result of the moment analysis of the NGC 3109 data. It can be seen that the $\mathrm{HI}$ extends over nearly $1^{\circ}$, more than 4 times the optical diameter $\left(D_{25}\right)$. The nearly parallel isovelocity contours, typical of a solid-body type rotation curve, are clearly visible, as well as the warp of the Hi disk in the outer parts. The velocity dispersion map shows very well the gradient of $\sigma$ from $15 \mathrm{~km} \mathrm{~s}^{-1}$ in the center down to $5 \mathrm{~km} \mathrm{~s}^{-1}$ at the edge of the disk.

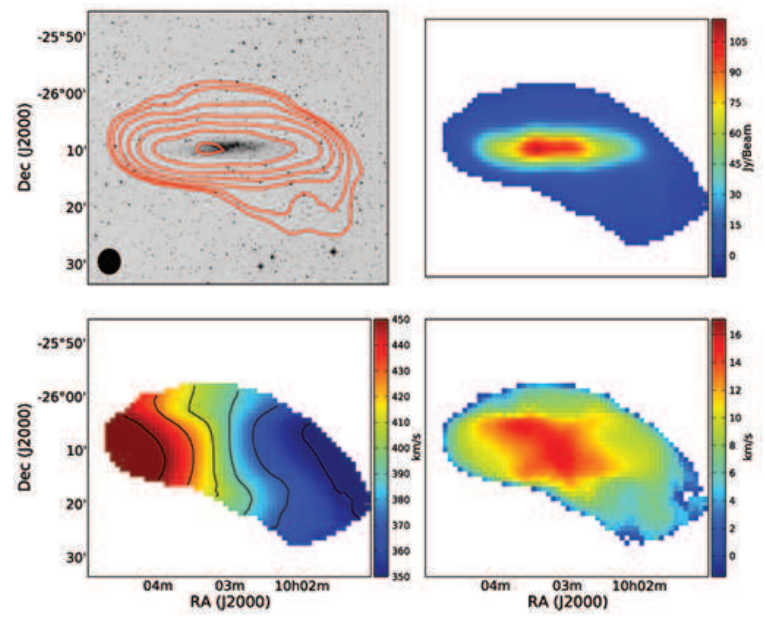

Fig. 3.- Moment maps of NGC 3109 from the KAT-7 na data cube. (top-left): total Hi emission map with the synthesize beam in the bottomleft corner. The contours are 0.4, 0.8, 1.6, 3.2, $6.4,12.8,25.6 \times 10^{20}$ atoms $\mathrm{cm}^{-2}$, superposed on a DSS B image. (top-right): total Hi emission map; (bottom-left): velocity field. The contours are $350,370,390,410,430,450 \mathrm{~km} \mathrm{~s}^{-1}$; (bottomright): velocity dispersion.

Fig. 4 gives the integrated Hi profile for NGC 3109. Profile widths of $\Delta V_{50}=118 \pm 3 \mathrm{~km} \mathrm{~s}^{-1}$ 
and $\Delta \mathrm{V}_{20}=136 \pm 3 \mathrm{~km} \mathrm{~s}^{-1}$ are derived. Since the $\mathrm{HI}$ distribution is clearly lopsided, with more gas on the approaching (SW) than on the receding side, we adopt the midpoint velocity of $404 \pm 2 \mathrm{~km}$ $\mathrm{s}^{-1}$ as more representative of the systemic velocity than the intensity-weighted mean velocity. This can be compared to $404 \mathrm{~km} \mathrm{~s}^{-1}, \Delta \mathrm{V}_{50}=123 \mathrm{~km}$ $\mathrm{s}^{-1}$ and $\Delta \mathrm{V}_{20}=137 \mathrm{~km} \mathrm{~s}^{-1}$ in Jobin \& Carignan (1990). An integrated flux of $1142 \pm 110 \mathrm{Jy} \mathrm{km} \mathrm{s}^{-1}$ is measured which corresponds at our adopted distance of $1.3 \mathrm{Mpc}$ to a $\mathrm{HI}$ mass of $\mathrm{M}_{\mathrm{HI}}=4.6 \times 10^{8}$ $\mathrm{M}_{\odot}$ for a $\mathrm{HI}$ mass-to-luminosity ratio $\mathrm{M}_{\mathrm{HI}} / \mathrm{L}_{B}$ of 1.0, showing the gas-rich nature of NGC 3109 . With a HPBW of the primary beam of nearly one degree and the short baselines available, no flux should be missed by these observations.

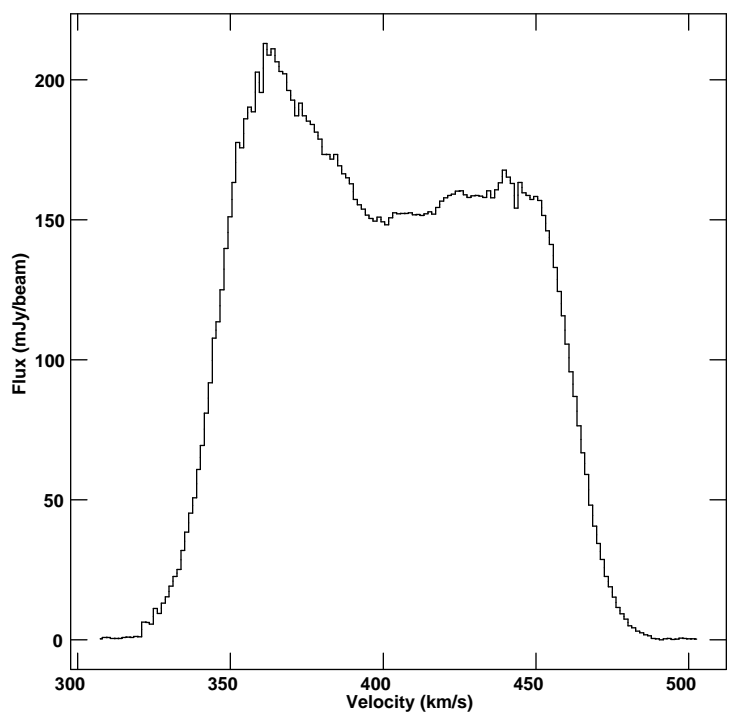

Fig. 4.- Global Hi line profile of NGC 3109 from the KAT-7 na data cube. The small dip $\sim 450$ $\mathrm{km} \mathrm{s}^{-1}$ is due to internally generated narrow band RFI.

Two methods of kinematical analysis will be used for the NGC 3109 data, namely an intensityweighted moment analysis and a Gauss-Hermite polynomial profile fit. With such high $\mathrm{S} / \mathrm{N}$ data and low velocity gradient RC, not much difference is expected between the two types of analysis. As a result, the analysis yielding the smaller errors and the largest radius $\mathrm{RC}$ will be used for the mass model analysis in Sec. 4. The RCs are derived in
Sec. 3.4

\subsection{VLA-ANGST data on NGC 3109 and Antlia}

NGC 3109 and Antlia were observed at the VLA as part of the VLA-ANGST survey (Ott et al. 2012). The galaxies were observed for $\sim 9$ hours in BnA, $\sim 3$ hours in $\mathrm{CnB}$ and $\sim 3$ hours in DnC configurations giving access to scales from $\sim 6$ " to $\sim 15$ '. The hybrid configurations were used to get more circular beams for these two southern objects. The data were gridded with two different weighting functions: na weighting for maximum sensitivity and ro weighting for maximum spatial resolution (smaller synthesized beam). We use the ro weighted maps for NGC 3109 and the na weighted maps for Antlia. The parameters of the VLA observations are given in Table 6 .

Table 6: Parameters of the VLA-ANGST observations.

\begin{tabular}{lcc}
\hline \hline Parameter & NGC 3109 & Antlia \\
\hline FWHM of primary beam & $31.5^{\prime}$ & $31.5^{\prime}$ \\
FWHM of synthesized beam & $7.6^{\prime \prime} \times 5.0^{\prime \prime}$ & $14.1^{\prime \prime} \times 13.9^{\prime \prime}$ \\
Total Bandwidth (MHz) & 1.56 & 0.78 \\
Number of channels & 256 & 128 \\
Central frequency $(\mathrm{MHz})$ & 1418.5 & 1418.6 \\
Channel width $\left(\mathrm{km} \mathrm{s}^{-1}\right)$ & 1.3 & 1.3 \\
RMS noise $(\mathrm{mJy} / \mathrm{beam})$ & 1.7 & 1.0 \\
Conversion ${ }^{\circ} \mathrm{K} /(\mathrm{mJy} /$ beam) & 15.8 & 3.06 \\
Maps gridding & $1.0^{\prime \prime} \times 1.0^{\prime \prime}$ & $1.5^{\prime \prime} \mathrm{x} 1.5^{\prime \prime}$ \\
Maps size & $2048^{2}$ & $1024^{2}$ \\
Weighting function & robust & natural \\
Phase calibrator & $0921-263$ & $0921-263$ \\
\hline
\end{tabular}

Figure 5 shows the velocity field obtained with the ANGST data for NGC 3109. The nearly parallel contours are again clearly visible. From the Hi emission map, we measure a total flux of 723 Jy $\mathrm{km} \mathrm{s}^{-1}$ which, at our adopted distance, corresponds to a $\mathrm{M}_{\mathrm{HI}}=2.9 \times 10^{8} \mathrm{M}_{\odot}$, which is nearly $40 \%$ less than the Hi detected with KAT -7 . Because the VLA is not sensitive to scales larger than $15^{\prime}$, the VLA-ANGST data will be missing some flux and their Hi mass will be clearly underestimated for NGC 3109. In fact, the Hi mass measured is exactly the same as that found by Jobin \& Carignan (1990) with a VLA mosaic of two fields. So the problem with the VLA data is 
not as much the smaller HPBW of the antennae but more the lack of short baselines.

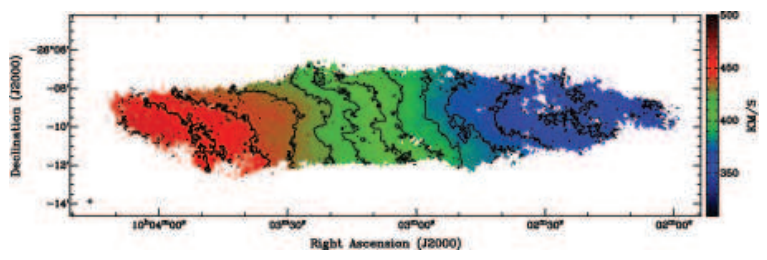

Fig. 5.- Velocity field for the ro-weighted VLAANGST velocity field.

The VLA-ANGST data, with its high spatial resolution, is much better suited to study the $\mathrm{HI}$ distribution in Antlia, since KAT-7 has barely two beamwidths across the object. Figure 6 gives the total Hi emission map for Antlia, where the lowest contour corresponds to $3.0 \times 10^{19}$ atoms $\mathrm{cm}^{-2}$. A total flux of $3.7 \pm 0.4 \mathrm{Jy} \mathrm{km} \mathrm{s}{ }^{-1}$ is found which, at an adopted distance of $1.31 \pm 0.03$ Mpc (Pimbblet \& Couch 2012), corresponds to a $\mathrm{M}_{\mathrm{HI}}=1.50 \pm 0.15 \times 10^{6} \mathrm{M}_{\odot}$. This time, most of the flux should have been detected since Antlia is much smaller than NGC 3109. However, this total flux is twice as much as that of the HIPASS data where Barnes \& de Blok (2001) found $1.7 \pm 0.1$ $\mathrm{Jy} \mathrm{km} \mathrm{s}^{-1}$ and still $40 \%$ more than the value of $2.7 \pm 0.5 \mathrm{Jy} \mathrm{km} \mathrm{s}^{-1}$ found by Fouqué et al. (1990). The reason for this large difference is not clear. But since we do not have access to the raw data, it is difficult for us to investigate further.

Figure 7 gives the integrated $\mathrm{HI}$ profile for Antlia. An integrated flux of $3.7 \pm 0.30 \mathrm{Jy} \mathrm{km}$ $\mathrm{s}^{-1}$ is found, which is similar to the flux derived from the Hi emission map. An intensity-weighted mean velocity of $360 \pm 2 \mathrm{~km} \mathrm{~s}^{-1}$ is derived along with $\Delta \mathrm{V}_{50}=23 \pm 3 \mathrm{~km} \mathrm{~s}^{-1}$ and $\Delta \mathrm{V}_{20}=33 \pm 3$ $\mathrm{km} \mathrm{s}^{-1}$. This can be compared to $362 \pm 2 \mathrm{~km} \mathrm{~s}^{-1}$ and $\Delta V_{20}=30 \pm 2 \mathrm{~km} \mathrm{~s}^{-1}$ for Barnes \& de Blok (2001) and $361 \pm 2 \mathrm{~km} \mathrm{~s}^{-1} \Delta \mathrm{V}_{50}=21 \pm 4 \mathrm{~km}$ $\mathrm{s}^{-1}$ and a $\Delta \mathrm{V}_{20}=33 \pm 5 \mathrm{~km} \mathrm{~s}^{-1}$ for Fouqué et al. (1990).

\subsection{Comparison of the different $\mathrm{HI}$ data sets}

The limiting surface densities of the different interferometric studies are given in Table 7 As far as the VLA data are concerned, one should not be surprised that the Jobin \& Carignan (1990) data

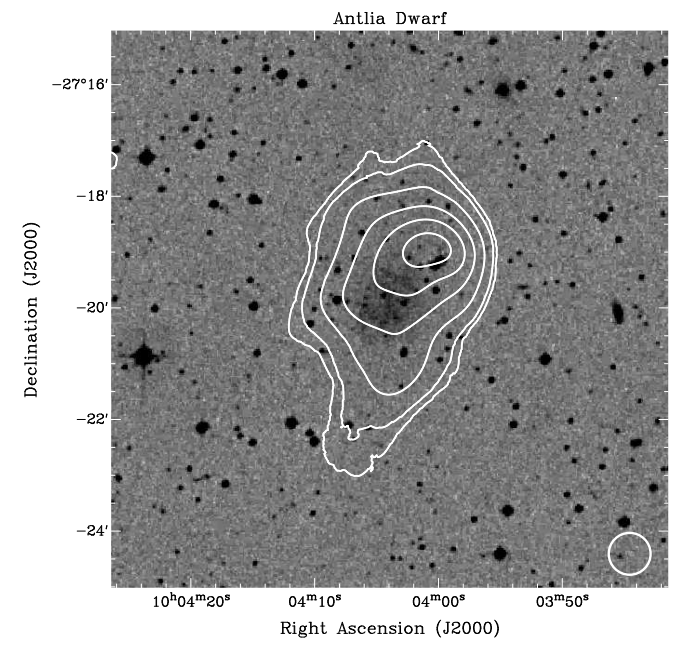

Fig. 6. - Total VLA-ANGST Hi emission map of Antlia. The na-weighted data have been spatially smoothed to $45^{\prime \prime}$ (see the beam in the bottom right corner). The contours are at $0.3,0.45,0.9,1.5,2.1 \& 2.7 \times 10^{20}$ atoms $\mathrm{cm}^{-2}$. The contours are superposed on a DSS B image.

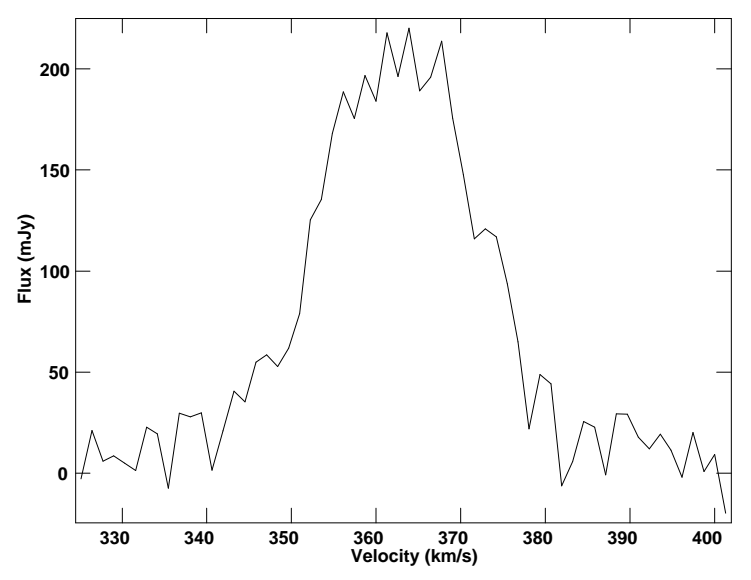

Fig. 7.- VLA-ANGST global Hi profile of Antlia using the na-weighted data smoothed to $45^{\prime \prime}$.

go deeper than the ANGST data since they are a mosaic of 2 fields with the same observing time in DnC configuration than the single field ANGST data. The $1.3 \mathrm{~km} \mathrm{~s}^{-1}$ resolution ANGST data is useful for comparisons with the Jobin \& Carignan (1990) $10.3 \mathrm{~km} \mathrm{~s}^{-1}$ resolution data in the inner regions of NGC 3109. But mainly, that data provide 
more information on the Hi distribution of Antlia, for which the KAT-7 data is of too low spatial resolution.

Table 7: Limiting Hi surface densities of the different interferometer studies for NGC 3109.

\begin{tabular}{lcc}
\hline \hline Reference & atoms cm & Hi size \\
\hline KAT-7 & $1.0 \times 10^{19}$ & $58^{\prime} \times 27^{\prime}$ \\
ANGST, VLA & $2.0 \times 10^{20}$ & $32^{\prime} \times 08^{\prime}$ \\
Barnes \& de Blok (2001) & $2.0 \times 10^{17}$ & $85^{\prime} \times 55^{\prime}$ \\
Jobin \& Carignan (1990) & $1.0 \times 10^{19}$ & $40^{\prime} \times 12^{\prime}$ \\
\hline
\end{tabular}

In view of the surface densities limits, we see that while both the KAT-7 and the Jobin \& Carignan (1990) reach $1.0 \times 10^{19} \mathrm{~cm}^{-2}$, the KAT-7 data covers a larger area since it is sensitive to large scales invisible to the VLA. As for the Barnes \& de Blok (2001) data reaching the much lower surface densities of $2.0 \times 10^{17} \mathrm{~cm}^{-2}$, they provide the largest detected size $\left(85^{\prime} \times 55^{\prime}\right)$. However, this increase in size may be due partly to the large $\sim 15.5^{\prime}$ HIPASS beam.

The HI mass estimates of both the single dish and the interferometric observations can be found in Table 8. The first thing to notice is the larger mass obtained by the single dish observations compared to the aperture synthesis ones, the only exception being the Whiteoak \& Gardner (1977) data which come from a single pointing of the Parkes $64 \mathrm{~m}$ radio telescope. With a $\sim 15^{\prime}$ beam, necessarily a lot of the flux extending over $\sim 1^{\circ}$ has been missed. For the others, the discrepancies can be explained by either the way the multi-pointing data have been combined or most likely that the correction for self-absorption that most of these authors have applied has been overestimated. No such correction has been applied to the synthesis data.

As for the synthesis data, we see that both sets of VLA data agree exactly. We would have expected some more flux from the deeper Jobin \& Carignan (1990) data but since most of the flux is in the bright central components, the difference is probably just of the order of the errors. On the other hand, both the KAT-7 data and the HIPASS data agree very well which is surely indicative that, in both cases, no flux is missed. Because both those data sets see all the scales, they detect nearly $40 \%$ more flux than the VLA, which do not have the proper short spacings
Table 8: Different HI mass estimates for NGC 3109*

\begin{tabular}{ll}
\hline \hline Reference & $10^{8} \mathrm{M}_{\odot}$ \\
\hline Aperture synthesis & \\
\hline KAT-7 & $4.6 \pm 0.5$ \\
ANGST, VLA & $2.9 \pm 0.3$ \\
Barnes \& de Blok $(2001)$ & $4.5 \pm 0.6$ \\
\hline Jobin \& Carignan (1990) & $2.9 \pm 0.6$ \\
\hline Single-dish & \\
\hline Huchtmeier et al. (1980) & $6.6 \pm 0.7$ \\
Whiteoak \& Gardner (1977) & $2.1 \pm 0.2$ \\
Dean \& Davies (1975) & $5.9 \pm 0.3$ \\
Huchtmeier (1973) & 5.9 \\
van Damme (1966) & 5.9 \\
\hline Epstein (1964) & $6.6 \pm 0.8$ \\
\hline
\end{tabular}

* Naturally, all the masses have been corrected to our adopted distance of $1.3 \mathrm{Mpc}$.

to see scales larger than $15^{\prime}$.

\subsection{Derivation of the optimal RC}

The same method is used to derive the $\mathrm{RC}$ for both the VLA-ANGST and the KAT-7 data sets. For this study, we used the implementation of the tilted ring model in the GIPSY task ROTCUR (Begeman 1989).

\subsubsection{Tilted Ring Models}

A set of concentric rings is used to describe the motion of the gas in the galaxy. The gas is assumed to be in circular motion. Each ring is characterized by a set of 5 orientation parameters, namely: a rotation centre $\left(x_{c}, y_{c}\right)$, a systemic velocity $V_{\text {sys }}$, an inclination $i$, a Position Angle $P A$ and by a rotation velocity $V_{C}$. Naturally, the rotation centre $\left(x_{c}, y_{c}\right)$ and the systemic velocity $V_{s y s}$ should be the same for all the rings but $i$ and $P A$ will vary if the HI disk is warped.

The line of sight velocity at any $(x, y)$ position in a ring with radius $R$ is given by

$$
V(x, y)=V_{\text {sys }}+V_{C} \sin (i) \cos (\theta)
$$

where $\theta$ is the position angle with respect to the receding major axis measured in the plane of the galaxy. $\theta$ is related to the actual $P A$ in the plane 
of the sky by

$$
\begin{aligned}
& \cos (\theta)=\frac{-\left(x-x_{0}\right) \sin (P A)+\left(y-y_{0}\right) \cos (P A)}{R} \\
& \sin (\theta)=\frac{-\left(x-x_{0}\right) \cos (P A)+\left(y-y_{0}\right) \cos (P A)}{R \cos (i)}
\end{aligned}
$$

A $|\cos \theta|$ weighting function and an exclusion angle of \pm 15 deg about the minor axis have been used to give maximum weight to the velocity points close to the major axis and minimize the influence of large deprojection errors close to the minor axis in view of the large inclination of the galaxy. The width of the rings has been matched to the synthesized beam size to make sure that the velocity points are independent.

The method consists at finding for each ring the best set of the 5 orientation parameters $\left(x_{c}, y_{c}\right)$, $V_{\text {sys }}, i$ and $P A$ which minimizes the dispersion of $V_{C}$ inside the ring. The following procedure is followed:

- The rotation center $\left(x_{c}, y_{c}\right)$ and the systemic velocity $V_{\text {sys }}$ are determined first by keeping $i$ and $P A$ fixed (usually using the optical values). The rotation center and the systemic velocity have to be determined simultaneously since they are correlated. They are searched using only the central rings (e.g. $R \leq R_{25}$ ) since there is usually no warp within the optical disk.

- Now, keeping $\left(x_{c}, y_{c}\right)$ and $V_{\text {sys }}$ fixed, $i$ and $P A$ are looked for to map the warp of the HI disk, which usually starts just outside the optical. Here also, $i$ and $P A$ have to be determined simultaneously since they are also correlated.

- The previous two steps were done using the data of all the galaxy. Using the same fixed $\left(x_{c}, y_{c}\right)$ and $V_{\text {sys }}$, the previous step is repeated for the approaching and receding sides separately to see possible departures from axisymmetry.

The errors on $V_{C}$ will be the quadratic sum of the dispersion $\sigma$ in each ring and half the difference between the approaching and the receding sides:

$$
\Delta V=\sqrt{\sigma^{2}(V)+\left(\frac{\left|V_{a p p}-V_{r e c}\right|}{2}\right)^{2}}
$$

Since the mass models assume an axisymmetric system, we think that this way of calculating the errors is more representative of the true uncertainties, when comparing the $\mathrm{RC}$ to the model.

\subsubsection{VLA-ANGST RC}

For the derivation of the kinematics of NGC 3109 , we use the ro-weighted VLA-ANGST data. We find that the rotation center is coincident with the optical center and derive a systemic velocity $V_{\text {sys }}=402 \mathrm{~km} \mathrm{~s}^{-1}$. By keeping those parameters fixed, we then fitted $i$ and $P A$. The solutions for the whole galaxy and separately for the approaching and receding sides are shown in Fig. 8, along with a comparison with Jobin \& Carignan (1990) in Fig. 9. We see that the warp which starts around the Holmberg radius $\left(R_{H O}\right)$, is more important on the approaching (SW) side and that the $\mathrm{RC}$ from both VLA datasets agree very well, despite the difference in velocity resolutions $(1.3 \mathrm{~km}$ $\mathrm{s}^{-1}$ vs $10.3 \mathrm{~km} \mathrm{~s}^{-1}$ ). Because Jobin \& Carignan (1990) provide asymmetric drift corrections, their $\mathrm{RC}$ will be used for the mass models in Sec. 4.

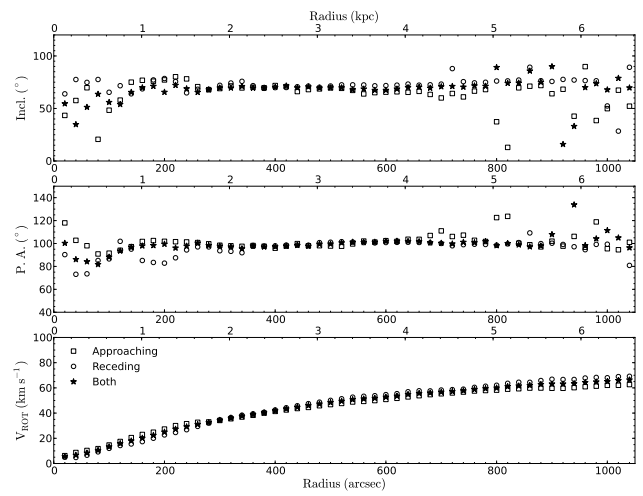

Fig. 8.- Tilted-ring model using the ro VLAANGST data for both sides and independently for the approaching and the receding side.

\subsubsection{KAT-7 RC}

Fig. 10 shows the tilted-ting model for the intensity-weighted moment analysis of the KAT7 na-weighted data of NGC 3109. We find $V_{\text {sys }}$ $=405 \pm 2 \mathrm{~km} \mathrm{~s}^{-1}, P A \sim 96^{\circ} \pm 4$ and $i \sim 61^{\circ}$ \pm 8 . The rotation center is found to be $\sim 0.5^{\prime}$ North from the optical center at $10^{\mathrm{h}} 03^{\mathrm{m}} 06.9^{\mathrm{s}}-$ 


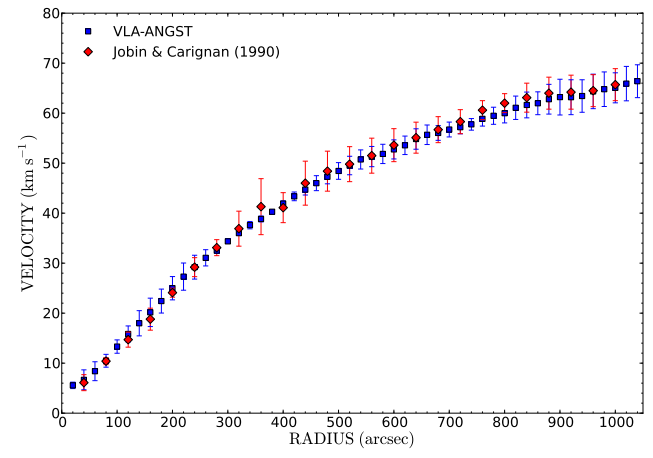

Fig. 9.- Comparison of the VLA-ANGST and the Jobin \& Carignan (1990) rotation curves.

$26^{\circ} 08^{\prime} 58^{\prime \prime}$. This offset from the optical center is not significant and could be due to the larger synthesized beam of KAT-7. It can be seen that the agreement between the approaching and receding sides is much better than for the VLA-ANGST data. The increase in sensitivity allows us to extend the RC out to $\sim 32^{\prime}(\sim 12 \mathrm{kpc})$. Despite the low spatial resolution, no real sign of beam smearing is seen when comparing this RC to the VLA data. This may not be surprising in view of the solid-body nature of the RC.

Fig. 11 shows the tilted-ting model for the Gauss-Hermite polynomials profile fitting analysis of the KAT-7 na-weighted data. The kinematical parameters found are very similar to those of the moment analysis with $V_{\text {sys }}=406 \mathrm{~km} \mathrm{~s}^{-1}, P A$ $\sim 97^{\circ}$ and $i \sim 61^{\circ}$ and the same rotation center. The Gauss-Hermite polynomials are fitted to the spectra in each pixel, where the peak of the fitted profile rises above 5 -sigma. As such, profiles from very faint emission at the edge of the galaxy are not strong enough to ensure a good fit. GaussHermite fits will therefore fail at large radii where the average signal-to-noise is lower than the cutoff. Lowering the cutoff produces too many bad velocity points. Therefore, since the RC using this technique is only defined out to $24^{\prime}$, the moment analysis RC is preferred for the mass models in Sec4.

\subsubsection{Asymmetric drift corrections}

In the case of a galaxy like NGC 3109, where the velocity dispersion represents a substantial frac-

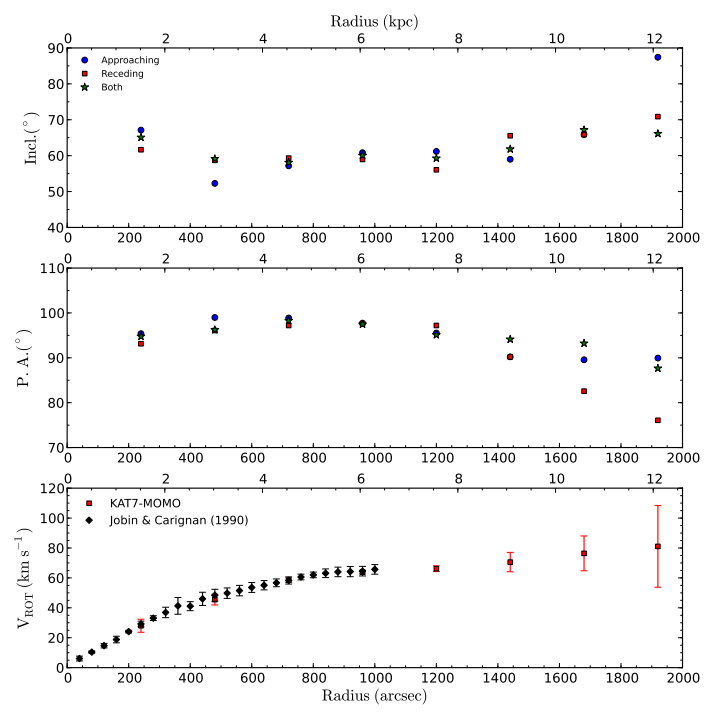

Fig. 10.- Tilted-ring model using the KAT-7 data derived from a moment analysis (intensity weighted) for both sides and independently for the approaching and the receding side and comparison with the RC of Jobin \& Carignan (1990).

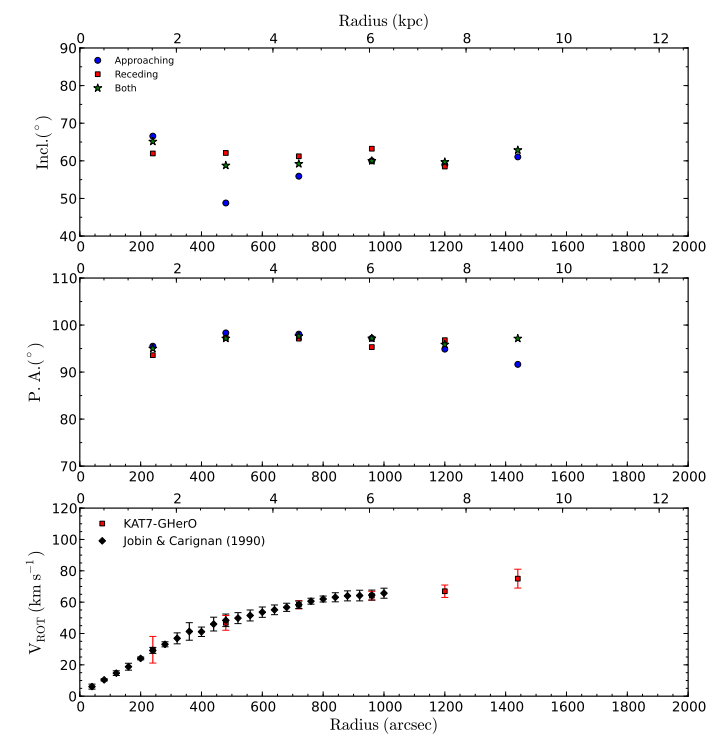

Fig. 11.- Tilted-ring model using the KAT7 data derived from a Gauss-Hermite polynomials analysis (profile fitting) for both sides and independently for the approaching and the receding side and comparison with the $\mathrm{RC}$ of Jobin \& Carignan (1990). 
tion of the rotational velocity $\left(\sigma / V_{\max } \geq 15-\right.$ $20 \%$ ) and thus provides part of the gravitational support, a correction for asymmetric drift must be applied. Following the procedure used by Côté. Carignan \& Freeman (2000), the corrected circular velocity is given by

$$
V_{c}^{2}=V_{o}^{2}-2 \sigma \frac{\delta \sigma}{\delta \ln R}-\sigma^{2} \frac{\delta \ln \Sigma}{\delta \ln R}
$$

where $V_{c}$ is the corrected velocity, $V_{o}$ is the observed one, $\sigma$ is the velocity dispersion and $\Sigma$ is the gas surface density. The asymmetric drift corrections are uncertain by about $25 \%$ (Lake \& Skillman 1989).

Table 9 shows the radial profiles for the surface densities and the velocity dispersion that were derived using the task IRING in AIPS. For the mass models, the Hi surface densities will be multiplied by $4 / 3$ to correct for Helium. The radial profiles will be used to correct for the asymmetric drift. Table 10 presents the corrected values: column (1) gives the radius in arcsec, column (2) the observed rotation velocities, column (3) the errors on those velocities, column (4) the ratio between the velocity dispersion and the circular velocity, column (5) gives the amplitude of the correction and column (6) the corrected velocities used for the mass models. As can be seen, when $\sigma / \mathrm{V}$ is greater than $20 \%$, the corrections can be of the order of a few $\mathrm{km} \mathrm{s}^{-1}$ which is nearly $10 \%$ for a slow rotator such as NGC 3109. Usually, when $\sigma / \mathrm{V}$ is less than $20 \%$, the corrections are of $1 \mathrm{~km}$ $\mathrm{s}^{-1}$ or less and usually neglected because they are smaller than the errors.

\section{Mass models analysis}

Low mass Surface Density (LSD) galaxies are galaxies whose mass profiles are dominated by dark matter (DM) at all galactocentric radii. LSD properties were first identified in dwarf Irregular (dIrr) galaxies (such as the prototype dIrr DDO 154: Carignan \& Freeman 1988; Carignan \& Purton 1998) and later in late-type dwarf spirals (see e.g. NGC 5585: Côté. Carignan \& Sancis 1991). Despite the uncertainties on the exact $\mathrm{M} / \mathrm{L}$ ratio of the luminous disk, LSD galaxies, such as NGC 3109, are clearly DM dominated at all radii. For that reason, they can be used to constrain important properties of dark matter haloes, such as the characteristic scale density
Table 9: Radial variation of the surface densities $\Sigma$ and of the velocity dispersion $\sigma$ for the KAT -7 data of NGC 3109 from the moment analysis.

\begin{tabular}{ccccc}
\hline $\begin{array}{c}\text { Radius } \\
(\operatorname{arcsec})\end{array}$ & $\begin{array}{c}\Sigma \\
\mathrm{M}_{\odot} \mathrm{pc}^{-2}\end{array}$ & $\begin{array}{c}\Delta \Sigma \\
\mathrm{M}_{\odot} \mathrm{pc}^{-2}\end{array}$ & $\begin{array}{c}\sigma \\
\left(\mathrm{km} \mathrm{s}^{-1}\right)\end{array}$ & $\begin{array}{c}\Delta \sigma \\
\left(\mathrm{km} \mathrm{s}^{-1}\right)\end{array}$ \\
\hline 160 & 6.03 & 0.38 & 15.31 & 0.06 \\
374 & 3.44 & 0.31 & 14.32 & 0.15 \\
608 & 2.00 & 0.21 & 12.35 & 0.24 \\
839 & 1.63 & 0.18 & 10.82 & 0.31 \\
1080 & 1.52 & 0.17 & 10.34 & 0.32 \\
1324 & 1.42 & 0.15 & 9.83 & 0.28 \\
1568 & 1.29 & 0.14 & 9.23 & 0.26 \\
1807 & 1.02 & 0.11 & 8.56 & 0.27 \\
2050 & 0.80 & 0.08 & 8.32 & 0.26 \\
2287 & 0.56 & 0.06 & 8.12 & 0.23 \\
2530 & 0.35 & 0.03 & 7.91 & 0.20 \\
2765 & 0.22 & 0.02 & 7.27 & 0.27 \\
\hline
\end{tabular}

Table 10: KAT-7 rotation curve of NGC 3109 from the moment analysis, corrected for asymmetric drift.

\begin{tabular}{cccccc}
\hline $\begin{array}{c}\text { Radius } \\
(\operatorname{arcsec})\end{array}$ & $\begin{array}{c}\mathrm{V}_{0} \\
\left(\mathrm{~km} \mathrm{~s}^{-1}\right)\end{array}$ & $\begin{array}{c}\Delta \mathrm{V} \\
\left(\mathrm{km} \mathrm{s}^{-1}\right)\end{array}$ & $\begin{array}{c}\sigma / \mathrm{V} \\
\%\end{array}$ & $\begin{array}{c}\mathrm{V}_{\text {corr }} \\
\left(\mathrm{km} \mathrm{s}^{-1}\right)\end{array}$ & $\begin{array}{c}\mathrm{V}_{\mathrm{C}} \\
\left(\mathrm{km} \mathrm{s}^{-1}\right)\end{array}$ \\
\hline 240 & 28.0 & 4.4 & 53 & 3.0 & 31.0 \\
480 & 45.5 & 3.6 & 29 & 3.3 & 48.8 \\
720 & 58.5 & 1.5 & 20 & 1.7 & 60.2 \\
960 & 63.9 & 1.2 & 17 & 1.0 & 64.9 \\
1200 & 66.2 & 1.9 & 15 & 0.6 & 66.8 \\
1440 & 70.6 & 6.5 & 14 & 0.8 & 71.4 \\
1680 & 76.4 & 11.6 & 12 & 1.4 & 77.8 \\
1920 & 81.1 & 27.3 & 10 & 0.6 & 81.7 \\
\hline
\end{tabular}

and radius, concentration, virial mass and the exact shape of the mass density profile. Ultimately, measuring the dark matter distribution of these galaxies is necessary if one wants to test the results obtained by numerical simulations of galaxy evolution in the framework of the Cold Dark Matter (CDM) paradigm (Navarro, Frenk \& White 1997) or test alternative gravity theories such as MOND (Milgrom 1983).

The study of their mass distribution has generated in the last 15 years the so-called cusp-core controversy: are rotation curves of LSD galaxies better reproduced by a cuspy halo as seen in the $\Lambda \mathrm{CDM}$ numerical simulations or by a halo with a nearly constant central density core as seen in most high spatial resolution observations 
(e.g. Blais-Ouellette, Amram \& Carignan 2001; de Blok, McGaugh \& Rubin 2001; Marchesini et al. 2002). A good review of this debate can be found in de Blok (2010).

Nowadays, galaxies are expected to form inside cuspy Cold Dark Matter halos. High resolution velocity fields have provided important observational constraints on the dark matter distribution in LSD galaxies. These two-dimensional data show clearly that dark matter-dominated galaxies tend to be more consistent with cored than cuspy halos, at odds with the theoretical expectations. So, a lot of efforts in the last few years has gone into identifying the physical processes that could have turned initially cuspy DM halos into cored ones (Governato et al. 2012; Pontzen \& Governato 2012; Kuzio de Naray \& Spekkens 2011; Kuzio de Narav et al. 2010; Governato et al. 2010). For example, one recently suggested solution to this problem is to enforce strong supernovae outflows that move large amounts of lowangular-momentum gas from the central parts and that pull on the central dark matter concentration to create a core (Famaey \& McGaugh 2013).

\subsection{Dark Matter models}

\subsubsection{The pseudo-isothermal DM model (ISO)}

The pseudo-isothermal DM halo is an observationally motivated model with a constant central density core. The density profile is given by:

$$
\rho_{I S O}(R)=\frac{\rho_{0}}{1+\left(\frac{R}{R_{c}}\right)^{2}}
$$

where $\rho_{0}$ is the central density and $R_{c}$ the core radius 1 . The corresponding rotation velocities are given by:

$$
V_{I S O}(R)=\sqrt{4 \pi G \rho_{0} R_{C}^{2}\left[1-\frac{R}{R_{C}} \arctan \left(\frac{R}{R_{C}}\right)\right]}
$$

We can describe the steepness of the inner slope of the mass density profile with a power law $\rho \sim$ $r^{\alpha}$. In the case of the ISO halo, where the inner density is an almost constant density core, $\alpha=0$.

\footnotetext{
${ }^{1}$ Note that it is different from the definition of $R_{c}$ for the true isothermal sphere (see King 1966; Carignan \& Freeman 1985).
}

\subsubsection{The Navarro, Frenk and White DM model} $(N F W)$

The NFW profile, also known as the "universal density profile" (Navarro, Frenk \& White 1997) is the commonly adopted dark matter halo profile in the context of the $\Lambda \mathrm{CDM}$ cosmology. It was derived from N-body simulations. The density profile is given by:

$$
\rho_{N F W}(R)=\frac{\rho_{i}}{R / R_{S}\left(1+R / R_{S}\right)^{2}}
$$

where $R_{S}$ is the characteristic radius of the halo and $\rho_{i}$ is related to the density of the universe at the time of collapse of the dark matter halo.The corresponding rotation velocities are given by:

$$
V_{N F W}(R)=V_{200} \sqrt{\frac{\ln (1+c x)-c x /(1+c x)}{x[\ln (1+c)-c /(1+c)]}}
$$

where $x=R / R_{200}$. It is characterized by a concentration parameter $c=R_{200} / R_{S}$ and a velocity $V_{200}$. The radius $R_{200}$ is the radius where the density contrast with respect to the critical density of the universe exceeds 200, roughly the virial radius (Navarro, Frenk \& White 1996). The characteristic velocity $V_{200}$ is the velocity at that radius. The NFW mass density profile is cuspy in the inner parts and can be represented by $\rho \sim r^{\alpha}$, where $\alpha=-1$.

\subsection{ISO \& NFW Models for NGC 3109}

Because of the way the different velocity points are weighted in the mass model fitting algorithm, we will not combine the high spatial resolution VLA data (Jobin \& Carignan 1990) with the low spatial resolution but high sensitivity KAT-7 data. Instead, we will run a set of models for each data set. The radial surface density profile of each data set will still be used, keeping in mind that the VLA data underestimate the Hi content, which will not be the case for the KAT-7 data. The I band luminosity profile of Jobin \& Carignan (1990) is preferred to IR (e.g. $2.6 \mu \mathrm{m}$ ) profiles because it extends to much larger radii.

NGC 3109 presents an interesting test for the DM models. Because it has no bulge and a very shallow velocity gradient, it is an ideal system to address the cusp-core controversy. The DM models are shown in Fig. 12 for the Jobin \& Carignan 
(1990) data and in Fig. 13 for the KAT-7 data. The results are summarized in Table 11, It can be seen that the ISO models fit almost perfectly the observed $\mathrm{RC}$ with a reduced $\chi^{2}$ of only 0.24 for the VLA data and 0.31 for the KAT-7 data. On the other hand, the NFW model has much less success with a reduced $\chi^{2}=12.9$ for the VLA data and 0.86 for the KAT-7 data. However, because of the low spatial resolution, the $\mathrm{KAT}-7$ data do not probe the very inner parts where the discrepancy with the observations is expected to be the greatest.
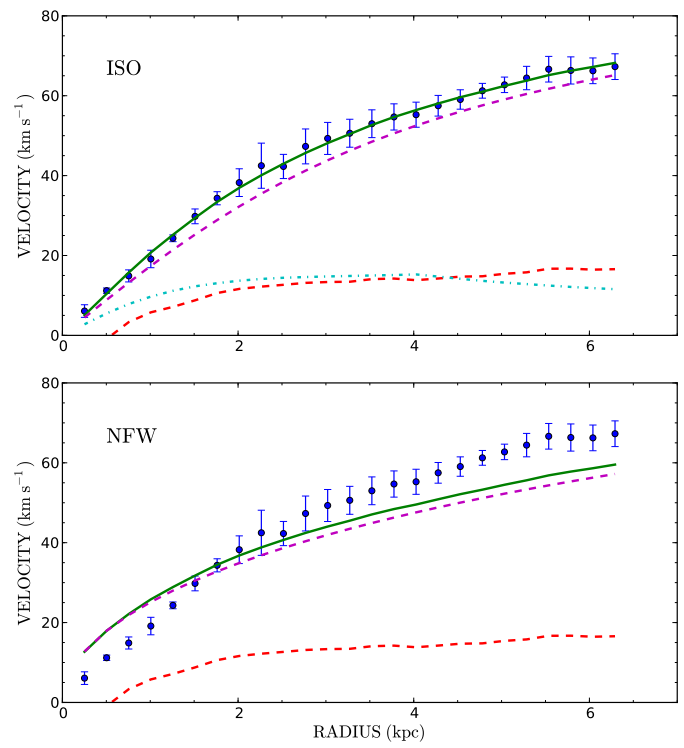

Fig. 12.- DM ISO (top) \& NFW (bottom) mass models for the observed VLA RC (blue points with errors) of NGC 3109 from Jobin \& Carignan (1990). The red dash curve is for the Hi disk, the dash-dot light blue curve is for the stellar disk, the purple dash curve is for the DM halo and the continuous green curve is the quadratic sum of the components. There is no disk component for the NFW model because the best-fit model yields $\mathrm{M} / \mathrm{L}=0$

The NFW model completely fails in the inner $1 \mathrm{kpc}$, overestimating the first velocity point by a factor of two for the VLA data. However, the main problem with the NFW models is that the best fits to both the VLA and the KAT-7 data suggest a $\mathrm{M} / \mathrm{L}$ of 0 for the stellar disk, which is unphysical. This is understandable since any stellar disk com-
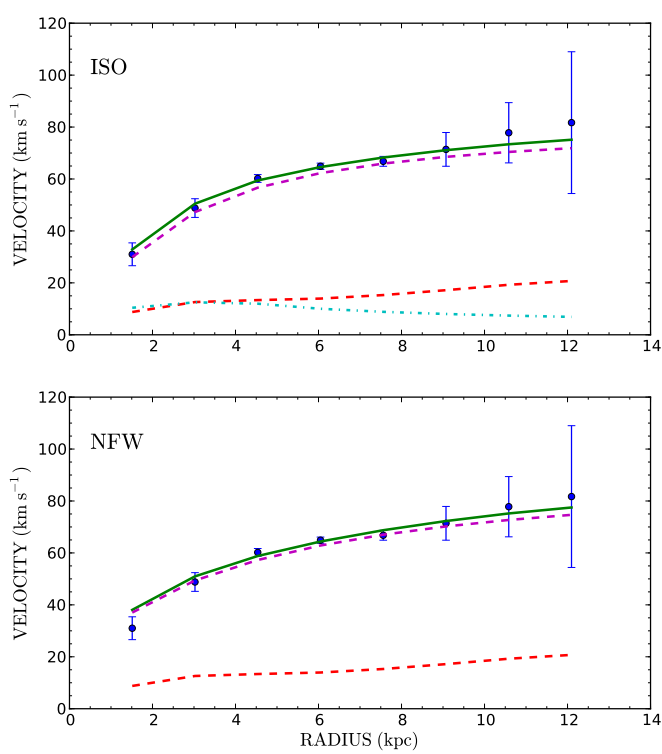

Fig. 13. - DM ISO (top) \& NFW (bottom) mass models for the observed $\mathrm{KAT}-7 \mathrm{RC}$ (blue points with errors) of NGC 3109. The symbols are the same as in Fig. 12

Table 11: Results for the DM mass models of NGC 3109 .

\begin{tabular}{|c|c|c|c|}
\hline Telescope & Model & Parameter & Result \\
\hline \multirow[t]{8}{*}{ VLA } & ISO & $(M / L)_{I}$ & 0.76 \\
\hline & & $R_{c}$ & $3.2 \mathrm{kpc}$ \\
\hline & & $\rho_{0}$ & $0.018 \mathrm{M}_{\odot} \mathrm{pc}^{-3}$ \\
\hline & & $\chi^{2}$ & 0.24 \\
\hline & NFW & $(M / L)_{I}$ & 0.00 \\
\hline & & $\mathrm{c}$ & 2.1 \\
\hline & & $R_{200}$ & $77.4 \mathrm{kpc}$ \\
\hline & & $\chi^{2}$ & 12.9 \\
\hline \multirow[t]{8}{*}{$\mathrm{KAT}-7$} & $\mathrm{ISO}$ & $(M / L)_{I}$ & 0.55 \\
\hline & & $R_{c}$ & $2.1 \mathrm{kpc}$ \\
\hline & & $\rho_{0}$ & $0.028 \mathrm{M}_{\odot} \mathrm{pc}^{-3}$ \\
\hline & & $\chi^{2}$ & 0.31 \\
\hline & NFW & $(M / L)_{I}$ & 0.00 \\
\hline & & $\mathrm{c}$ & 3.9 \\
\hline & & $\mathrm{R}_{200}$ & $58.1 \mathrm{kpc}$ \\
\hline & & $\chi^{2}$ & 0.86 \\
\hline
\end{tabular}

ponent would just increase the discrepancy in the inner parts. On the other hand, the best fit ISO model has $(\mathrm{M} / \mathrm{L})_{I}=0.55-0.76$ which is quite compatible with the value predicted by stellar popula- 
tion models of $0.67 \pm 0.04$ (Bell \& de Jong 2001) for the $I$ band. Without any doubt, in the DM halo paradigm, those results confirm that NGC 3109 has a cored and not a cuspy halo.

\subsection{MOND models}

MOND was proposed by Milgrom (1983) as an alternative to dark matter. Milgrom postulated that at small accelerations the usual Newtonian dynamics break down and the law of gravity needs to be modified. MOND has been claimed to be able to explain the mass discrepancies in galaxies without dark matter (e.g. Begeman. Broeils \& Sanders 1991; Sanders 1996; Bottema et al. 2002). Therefore, in the MOND formalism, only the contributions of the gas component and of the stellar component are required to explain the observed rotation curves.

The transition between the Newtonian and the MONDian regime is characterized by an acceleration threshold value called $\mathrm{a}_{0}$ below which MOND should be used. So, in the MOND framework, the gravitational acceleration of a test particle is given by :

$$
\mu\left(x=g / a_{0}\right) g=g_{N}
$$

where $g$ is the gravitational acceleration, $\mathrm{a}_{0}$ is a new universal constant which should be the same for all galaxies, $\mu(\mathrm{x})$ is the MOND interpolating function and $g_{N}$ the Newtonian acceleration.

The standard and simple interpolating functions are mostly used in the literature. The standard $\mu$-function is the original form of the interpolating function proposed by Milgrom (1983). However, Zhao \& Famaey (2006) found that a simplified form of the interpolating function not only provides also good fits to the observed rotation curves but the derived mass-to-light ratios are more compatible with those obtained from stellar populations synthesis models.

\subsubsection{MOND models using the "standard" inter- polation function}

The standard interpolating function is given as

$$
\mu(x)=\frac{x}{\sqrt{1+x^{2}}}
$$

For $x \ll 1$ the system is in deep MOND regime with $g=\left(g_{N} \mathrm{a}_{0}\right)^{1 / 2}$ and for $x \gg 1$ the gravity is
Newtonian.

The MOND rotation curve becomes:

$$
V_{\text {rot }}^{2}=\frac{V_{\text {sum }}^{2}}{\sqrt{2}} \sqrt{1+\sqrt{1+\left(2 r a_{0} / V_{\text {sum }}^{2}\right)^{2}}}
$$

where

$$
V_{\text {sum }}^{2}=V_{b}^{2}+V_{d}^{2}+V_{g}^{2}
$$

$V_{b}, V_{d}, V_{g}$ are the contributions from the bulge, the disk and the gas to the rotation curve. In the case of the Magellanic-type spiral NGC 3109, there is no bulge to consider.

\subsubsection{MOND models using the "simple" inter- polation function}

The simple interpolating function is given by

$$
\mu(x)=\frac{x}{1+x}
$$

Using the same procedure as in previous section we can easily obtain the corresponding rotation velocities:

$V_{\text {rot }}^{2}=\sqrt{V_{b}^{2}+V_{d}^{2}+V_{g}^{2}} * \sqrt{a_{0} * r+V_{b}^{2}+V_{d}^{2}+V_{g}^{2}}$

\subsection{MOND Models for NGC 3109}

NGC 3109 presents an interesting test for MOND. As we have seen, it is close enough to have a very accurate distance determination using Cepheids and the largest portion of the luminous mass is in the form of gas and not stars, partly freeing us from the uncertainties due to the M/L value used for the disk. The internal accelerations are very low, therefore the galaxy is completely within the MOND regime (Lake 1989).

The MOND mass models for the VLA data are presented in Fig. 14 \& Table 12 and in Fig. 15 \& Table 13 for the KAT-7 data, for both the standard value of $a_{0}$, namely $1.21 \times 10^{-8} \mathrm{~cm} \mathrm{sec}^{-2}$ (Begeman. Broeils \& Sanders 1991) and with $a_{0}$ left as a free parameter. The standard and the simple interpolation functions are also illustrated.

In the case of the VLA data, the fits are poor for all the cases: they overestimate the velocities in the inner parts and they underestimate them in the outer parts. The reduced $\chi^{2}$ are much larger 

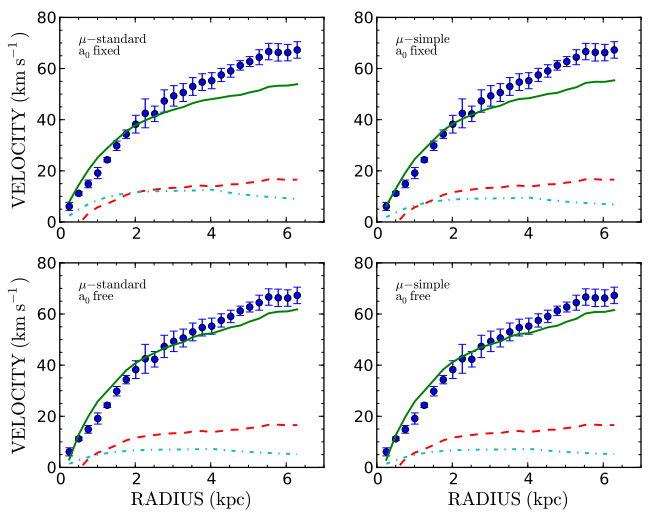

Fig. 14.- MOND mass models with $a_{0}$ fixed (top) and $a_{0}$ free (bottom) and for the standard (left) and the simple (right) interpolation functions for NGC 3109 using the RC of Jobin \& Carignan (1990). The red dash curve is for the HI disk, the dash-dot light blue curve is for the stellar disk, and the continuous green curve is the MOND model.

Table 12: Results for the MOND models of NGC 3109 for the VLA data.

\begin{tabular}{|c|c|c|c|}
\hline \multicolumn{4}{|c|}{$10^{-8} \mathrm{~cm} \mathrm{~s}^{-2}$} \\
\hline \multirow[t]{6}{*}{ fixed } & standard & $(\mathrm{M} / \mathrm{L})_{\mathrm{I}}$ & 0.45 \\
\hline & & $\mathrm{a}_{0}$ & 1.21 \\
\hline & & $\chi^{2}$ & 12.01 \\
\hline & simple & $(\mathrm{M} / \mathrm{L})_{\mathrm{I}}$ & 0.26 \\
\hline & & $a_{0}$ & 1.21 \\
\hline & & $\chi^{2}$ & 9.25 \\
\hline \multirow[t]{6}{*}{ free } & standard & $(\mathrm{M} / \mathrm{L})_{\mathrm{I}}$ & 0.15 \\
\hline & & $a_{0}$ & 2.48 \\
\hline & & $\chi^{2}$ & 5.50 \\
\hline & simple & $(\mathrm{M} / \mathrm{L})_{\mathrm{I}}$ & 0.15 \\
\hline & & $a_{0}$ & 2.07 \\
\hline & & $\chi^{2}$ & 5.57 \\
\hline
\end{tabular}

than for the DM fits, varying from 5.5 to 12 . However, since most of the luminous mass is in gas and since the VLA misses $40 \%$ of the flux, we should not expect good fits.

The MOND models have more significance for the KAT-7 data than for the VLA data, since we probe the gas on all scales. In this case we see that for the standard fixed value of $\mathrm{a}_{0}=1.21 \times 10^{-8}$

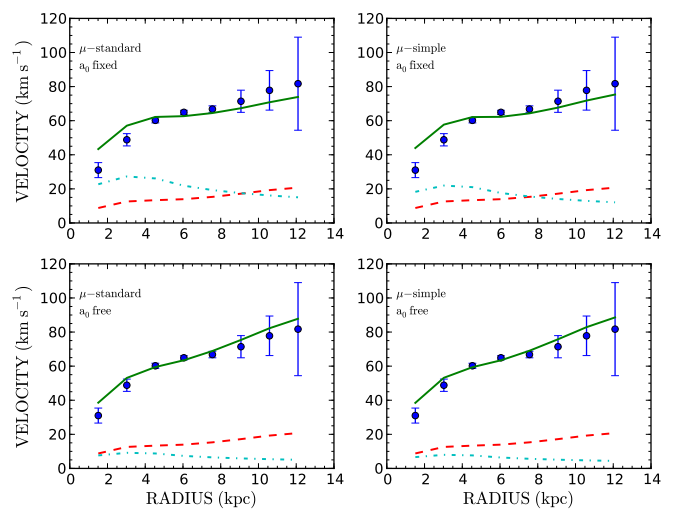

Fig. 15.- MOND mass models with $a_{0}$ fixed (top) and $a_{0}$ free (bottom) and for the standard (left) and the simple (right) interpolation functions for NGC 3109 using the RC from KAT-7 data. The symbols are the same as in Fig. 14

Table 13: Results for the MOND models of NGC 3109 for the KAT -7 data.

\begin{tabular}{cccc}
\hline \hline $\begin{array}{c}\mathrm{a}_{0} \\
10^{-8} \mathrm{~cm} \mathrm{~s}^{-2}\end{array}$ & $\mu$ & Parameter & Result \\
\hline fixed & standard & $(\mathrm{M} / \mathrm{L})_{\mathrm{I}}$ & 2.61 \\
& & $\mathrm{a}_{0}$ & 1.21 \\
& & $\chi^{2}$ & 2.96 \\
\cline { 2 - 4 } free & simple & $(\mathrm{M} / \mathrm{L})_{\mathrm{I}}$ & 1.69 \\
& & $\mathrm{a}_{0}$ & 1.21 \\
& & $\chi^{2}$ & 3.38 \\
\cline { 2 - 4 } & & & \\
& & $(\mathrm{M} / \mathrm{L})_{\mathrm{I}}$ & 0.29 \\
& & $\mathrm{a}_{0}$ & 3.48 \\
& & $\chi^{2}$ & 1.30 \\
\cline { 2 - 4 } & & $(\mathrm{M} / \mathrm{L})_{\mathrm{I}}$ & 0.22 \\
& & $\mathrm{a}_{0}$ & 3.25 \\
& & $\chi^{2}$ & 1.37 \\
\hline
\end{tabular}

$\mathrm{cm} \mathrm{sec}^{-2}$, the results of the models are poor with reduced $\chi^{2}$ of 3.0 to 3.4 and $(\mathrm{M} / \mathrm{L})_{B}$ values 3 to 4 times larger than the value predicted by stellar population models. However, we see that if $a_{0}$ is free to vary, we get more reasonable fits with reduced $\chi^{2}$ down to 1.30 and 1.37 , still $4-5$ times larger than for the DM ISO models. The main problems with those models are the very small $(\mathrm{M} / \mathrm{L})_{I}$ values, 2 to 3 times smaller than the expected values and the large values of the constant $a_{0}$ which is greater than a factor of 2 compared to the standard value. 


\section{Discussion}

We will now examine different questions that were raised by this study. The first one is the difference found between the kinematic inclination and the photometric values. This is an important point to understand since $i$ scales the $\mathrm{RC}$ and is responsible for the velocity gradient of the rising part of the RC, which really constrains the mass models and set the $\mathrm{M} / \mathrm{L}$ ratio of the luminous disk. Both tilted-ring models using the intensityweighted and the profile fitting techniques find the same inclination of $i=61^{\circ}$. It is important to understand how this parameter is determined. Assuming an axisymmetric disk, the tilted-ring model tries to find the value that minimizes the dispersion of the velocities in the rings.

On the other hand, looking at Table 14, it can be seen that the inclination varies from $78.5^{\circ}$ in $\mathrm{B}$ to $73.5^{\circ}$ in I and $69.5^{\circ}$ in the $3.6 \mu \mathrm{m}$. What is going on is quite clear. Each photometric band samples different stellar populations. The B-band samples the young blue Pop I stars which are confined to the thin disk, the I-band samples a mixture of young and old Pop I stars which are in a thicker disk while the $3.6 \mu \mathrm{m}$ is completely dominated by an old disk population in an even thicker disk. This explains that progression of the inclination from $\sim 80^{\circ}$ to $\sim 70^{\circ}$.

It is instructive to look at the ellipse fits to the Hi isophotes. As can be seen in Table 15, NGC 3109 is really composed of 2 disks: an inner one, corresponding to the optical disk, with $i \sim 76^{\circ}$, which is the mean of the $\mathrm{B}$ and I inclinations and an outer disk with $i \sim 63^{\circ}$ which, within the errors, agree with the kinematical inclination. The double disk is quite apparent when looking at the HI isophotes in Fig. 2. Therefore, since the photometric inclination is most sensitive to the emission of the stellar populations being traced, it is quite clear that the kinematic inclination should be preferred. The photometric values should only be used as starting values in the tilted-ring modeling.

Another point worth discussing is beam-smearing. With such a large synthesized beam $\sim 4^{\prime}$, one would have expected beam smearing to be quite important. However when looking at Figures 10 and 11. this does not seem to be the case. As explained in Carignan (1985), beam smearing is
Table 14: Optical orientation parameters of NGC 3109.

\begin{tabular}{lcc}
\hline \hline Parameter & & Ref \\
\hline Orientation parameters, $B$ & & \\
Axis ratio, b/a & $0.20 \pm 0.02$ & $(1)$ \\
Inclination* & $78.5^{\circ} \pm 2.0^{\circ}$ & $(1)$ \\
$P . A$. & $91.5^{\circ} \pm 1.0^{\circ}$ & $(1)$ \\
Orientation parameters, $I$ & & \\
Axis ratio, b/a & $0.28 \pm 0.02$ & $(2)$ \\
Inclination* & $73.5^{\circ} \pm 1.5^{\circ}$ & $(2)$ \\
$P . A$. & $93.0^{\circ} \pm 2.0^{\circ}$ & $(2)$ \\
Orientation parameters, $3.6 \mu \mathrm{m}$ & & \\
Axis ratio, b/a & $0.35 \pm 0.02$ & $(3)$ \\
Inclination* & $69.5^{\circ} \pm 1.5^{\circ}$ & $(3)$ \\
$P . A$. & $95.0^{\circ} \pm 2.0^{\circ}$ & $(3)$ \\
\hline
\end{tabular}

*no correction for intrinsic flattening.

References. - (1) Carignan (1985);

(2) Jobin \& Carignan (1990); (3) this study.

Table 15: Fits to the Hi isophotes.

\begin{tabular}{ccccc}
\hline \hline Isophotes & $\mathrm{q}$ (axis ratio) & $\Delta \mathrm{q}$ & $\mathrm{i}$ (inclination) & $\Delta \mathrm{i}$ \\
\hline 1.00 & 0.506 & 0.049 & 59.6 & 3.3 \\
3.00 & 0.403 & 0.022 & 66.2 & 1.4 \\
5.00 & 0.256 & 0.029 & 75.2 & 1.8 \\
7.00 & 0.246 & 0.018 & 75.8 & 1.1 \\
9.00 & 0.257 & 0.011 & 75.1 & 0.7 \\
11.00 & 0.255 & 0.007 & 75.2 & 0.3 \\
13.00 & 0.247 & 0.005 & 75.7 & 0.3 \\
15.00 & 0.247 & 0.004 & 75.7 & 0.2 \\
17.00 & 0.255 & 0.004 & 75.2 & 0.2 \\
19.00 & 0.278 & 0.005 & 73.9 & 0.3 \\
21.00 & 0.326 & 0.006 & 71.0 & 0.4 \\
23.00 & 0.448 & 0.011 & 63.4 & 0.7 \\
25.00 & 0.460 & 0.016 & 62.6 & 1.0 \\
27.00 & 0.460 & 0.018 & 62.6 & 1.2 \\
29.00 & 0.446 & 0.018 & 63.5 & 1.2 \\
31.00 & 0.446 & 0.010 & 63.5 & 0.6 \\
\hline
\end{tabular}

the result of the convolution of the Gaussian beam with the Hi distribution and the velocity gradient across the beam. If the Hi distribution is steep and/or the velocity gradient is large, the net effect will be to underestimate the observed velocity or conversely to overestimate the effective radius (the product of the convolution) that is observed. In 
the case of NGC 3109, the HI distribution is fairly flat across the beam and the velocity gradient of that galaxy is very small. Those two properties render the beam smearing negligible. Should we be observing another galaxy with a step velocity gradient and/or a steep radial Hi distribution, beam smearing would be important.

Let us now turn to the mass models. First, the results of the DM ISO models for both RCs confirm the previous results that it provides an almost perfect fit to the observed kinematics with the difference that the KAT- 7 model has a more massive Hi disk. As expected, this translates in a smaller stellar disk M/L value for the KAT-7 data. In both cases, the mass-to-light ratio found for the disk is compatible with population synthesis models. The NFW models again fail to reproduce the kinematics. In the inner kpc, the velocities are overestimated by a large factor, despite an unphysical M/L ratio of 0 for the stellar disk. In the DM halo paradigm, clearly NGC 3109 has a cored and not a cuspy halo, at least at the present epoch.

What about the MOND models ? Twenty-five years ago, Sanders (1986) pointed out that the MOND mass of NGC 3109 predicted using Milgrom's suggested value for $a_{0}$ was $5 \times 10^{8} \mathrm{M}_{\odot}$, thus larger than the Hi mass using the larger distance estimates known at the time between 1.7 to 2.6 Mpc (Carignan 1985). This is not the case anymore with the smaller well determined distance of $1.3 \mathrm{Mpc}$, but still our determined Hi mass with KAT -7 is less than $10 \%$ smaller than this MOND mass, which implies unphysically small $\mathrm{M} / \mathrm{L}$ values for the stars in the case where the fits have been improved letting the constant $a_{0}$ free to vary.

When Begeman. Broeils \& Sanders (1991) produced a MOND model using the Jobin \& Carignan (1990) VLA data, they argued that they could not get a good fit because the VLA data was missing a substantial part of the flux and that the HI mass had to be multiplied by 1.67 to get a reasonable fit. However, since the KAT -7 data retrieve all the NGC 3109 flux, this cannot be used as an argument with the present data which convincingly show that MOND cannot reproduce the observed kinematics of NGC 3109 with physically acceptable parameter values. Unless some other explanation can be found, the KAT -7 data surely challenge the MOND theory.
What about the possible interaction between the Magellanic-type spiral NGC 3109 and the dIrr/dSph Antlia, suggested by the Hi isophotes being slightly elongated? The high surface brightness sensitivity of the KAT-7 observations allow us to trace the lopsidedness of NGC 3109 to larger radii, however there is no further obvious evidence of an interaction with Antlia, leaving the question open for further investigation. NGC 3109 has a systemic velocity of $404 \mathrm{~km} \mathrm{~s}^{-1}$ while Antlia has $360 \mathrm{~km} \mathrm{~s}^{-1}$. Aparicio et al. (1997) calculated the physical separation between NGC 3109 and Antlia to be between 29 and $180 \mathrm{kpc}$, with a maximum separation of $37 \mathrm{kpc}$ for the pair to be bound.

The situation for the Local Group dwarfs can guide us. Grcevich \& Putman (2009) showed clearly that the majority of dwarf galaxies within $270 \mathrm{kpc}$ of the Milky Way or Andromeda are undetected in $\mathrm{HI}\left(<10^{4} \mathrm{M}_{\odot}\right.$ for the Milky Way dwarfs), while those further than $270 \mathrm{kpc}$ are predominantly detected with masses $10^{5}$ to $10^{8} \mathrm{M}_{\odot}$ (Antlia has an $\mathrm{HI}$ mass of $\sim 10^{6} \mathrm{M}_{\odot}$ ) meaning the nearby ones must have been stripped of their gas. While the halo of NGC 3109 is not as large as the Milky Way halo, if a close encounter had happened in the past, it is quite likely that Antlia would also have been stripped of its gas.

Nevertheless, some kind of interaction really seems to have taken place, as shown by the HI isophotes of the two galaxies pointing toward each other. However, in view of the large difference in masses between the two systems and the absence of any external traces of such an interaction, it is believed that the internal kinematics of NGC 3109 cannot have been severely altered.

Anyway, if such an interaction did take place, it would be more the rule than the exception. Massive galaxies usually have a significant population of gas-rich dwarf companions and interaction with these will show kinematic and morphological signatures in the extended HI disks (Mihos et al. 2012) such as warps, plumes, tidal tails, high-velocity clouds (e.g. Hibbard et al. 2001; Sancisi et al. 2008) or even stellar streams (Lewis et al. 2013). Galaxies are said to be embedded in the cosmic web, seen in the $\Lambda$ CDM numerical simulations Springel. Frenk \&White 2006). Such signatures of interaction have been studied close to massive galaxies such as the interconnecting network in the M81/M82 system 
(Yun. Ho \& Lo 1994) or the tidal tail in the Leo triplet (Havnes, Giovanelli \& Roberts 1979). More recently, Mihos et al. (2012) using the Green Bank Telescope (GBT) found a plume in the outer disk of M101 with a peak column density of $5 \mathrm{x}$ $10^{17} \mathrm{~cm}^{-2}$ and two new Hi clouds close to that plume with masses of $\sim 10^{7} \mathrm{M}_{\odot}$. While KAT-7 would not have detected such low column densities, it is interesting to look at our detection cloud mass limit.

To calculate a characteristic HI mass sensitivity, we assume that low-mass clouds would be unresolved in our beam and use the relation (Mihos et al. 2012):

$$
\left(\frac{\sigma_{M}}{M \odot}\right)=2.36 \times 10^{5}\left(\frac{D^{2}}{\mathrm{Mpc}}\right)\left(\frac{\sigma_{s}}{\mathrm{Jy}}\right)\left(\frac{\Delta V}{\mathrm{~km} \mathrm{~s}^{-1}}\right)
$$

where $D$ is the distance, $\sigma_{s}$ is the rms noise in one channel and $\Delta V$ is the channel width. This means that our $3 \sigma$ cloud mass detection limit is around $5-6 \times 10^{3} \mathrm{M}_{\odot}$ at the distance of NGC 3109 . We should thus have easily detected similar clouds like the ones observed around M101, if they had been present.

\section{Summary and conclusions}

The first Hi spectral line observations with the prototype radio telescope $\mathrm{KAT}-7$ have been presented. The high sensitivity of $\mathrm{KAT}-7$ to large scale, low column density emission comes not only from its compact configuration, but also from its very low $\mathrm{T}_{\text {sys }}$ receivers. With $\sim 25$ hours of observations per pointing, surface densities of $1.0 \mathrm{x}$ $10^{19}$ atoms $\mathrm{cm}^{-2}$ were reached, which could be improved when the telescope will be fully commissioned, since the theoretical noise was not reached with the present dataset.

The main results from this study are:

- A total Hi mass of $4.6 \times 10^{8} \mathrm{M}_{\odot}$ is measured for NGC 3109, using our adopted distance of $1.3 \mathrm{Mpc}$. This Hi mass, which is $\sim 40 \%$ larger than the values calculated using VLA observations, is surely a better estimate of the total HI mass of NGC 3109 since KAT7 is sensitive to the large scales for which the VLA is not.

- The Hi disk extends over a region of $58^{\prime}(\mathrm{EW})$ x $27^{\prime}(\mathrm{NS})$ down to a limiting column density of $1.0 \times 10^{19}$ atoms $\mathrm{cm}^{-2}$.

- The HI distribution is lopsided with more HI on the SW approaching side. Because of this, no intensity-weighted velocity is derived from the global profile but rather a mid-point velocity of $404 \pm 2 \mathrm{~km} \mathrm{~s}^{-1}$, believed to be more representative of the systemic velocity of NGC 3109. Profile widths of $\Delta \mathrm{V}_{50}=118 \pm 4 \mathrm{~km} \mathrm{~s}^{-1}$ and $\Delta \mathrm{V}_{20}=$ $133 \pm 3 \mathrm{~km} \mathrm{~s}^{-1}$ are derived.

- VLA-ANGST data were used to derive the HI properties of the dSph/dIrr Antlia, since the spatial resolution of $\mathrm{KAT}-7$ is too low to study this dwarf system. A total Hi mass of $1.5 \times 10^{6} \mathrm{M}_{\odot}$ is measured for our adopted distance of $1.31 \mathrm{Mpc}$. An intensity-weighted mean velocity of $360 \pm 2 \mathrm{~km} \mathrm{~s}^{-1}$ is derived along with a $\Delta \mathrm{V}_{50}=23 \pm 3 \mathrm{~km} \mathrm{~s}^{-1}$ and a $\Delta \mathrm{V}_{20}=33 \pm 3 \mathrm{~km} \mathrm{~s}^{-1}$.

- A tilted-ring model was obtained for the high velocity resolution VLA-ANGST data of NGC 3109. As on the Hi total intensity map, it can be seen that the warp is more important on the approaching side. The RC found is nearly identical to the one derived by Jobin \& Carignan (1990) which had a velocity resolution of $\sim 10 \mathrm{~km} \mathrm{~s}^{-1}$. Because the latter study provides correction for asymmetric drift, it was preferred for the mass model analysis.

- Rotation curves from the KAT-7 data of NGC 3109 were derived from two different types of analysis: an intensity-weighted moment analysis and a Gauss-Hermite polynomial profile fitting. Both data sets give essentially the same result with $V_{\text {sys }}=405 \mathrm{~km}$ $\mathrm{s}^{-1}$ and mean $P A=96^{\circ}$ and $i=61^{\circ}$, with very little difference between the approaching and the receding side. The $\mathrm{KAT}-7 \mathrm{RCs}$ agree very well with the VLA data in the inner parts while allowing to extend the rotation data by a factor of 2 out to $32^{\prime}$. Since the moment analysis data allow us to derive the RC further out, it was used for the mass models.

- The observationally motivated DM ISO model reproduces very well the observed 
RCs of both the Jobin \& Carignan (1990) and the KAT- 7 data while a cosmologically motivated NFW model gives a much poorer fit, especially in the very inner parts. Because of the high spatial and velocity resolutions data available and the very small errors on those velocities, this cannot be attributed to poor data. NGC 3109 definitely has a cored and not a cuspy DM halo.

- While it is clear that having the proper gas distribution has reduced the discrepancies between the observed $\mathrm{RC}$ and the MOND models, the unreasonable (M/L) and large $a_{0}$ values obtained lead us to conclude that we cannot get acceptable MOND models for NGC 3109. The distance being so well determined with very small errors from Cepheids observations and the HI mass so well constrained by the KAT -7 observations, uncertainties on these two values cannot explain why the MOND models fail to reproduce the observed kinematics with reasonable parameters.

- Besides some elongation of the outer isophotes, already seen in previous observations, no further evidence is found for past encounter and/or interaction between the Magellanictype spiral NGC 3109 and the dSph/dIrr Antlia. testbed for MeerKAT and the SKA such that any scientific result that can be obtained is a bonus. While most of the extragalactic Hi sources would be unresolved by the $\sim 4^{\prime}$ synthesized beam, many projects such as this one on NGC 3109 can be done on nearby very extended objects such as Local Group galaxies or galaxies in nearby groups like Sculptor.

We thank all the team of SKA South Africa for allowing us to get scientific data during the commissioning phase of $\mathrm{KAT}-7$ and are grateful to the ANGST team for making their reduced VLA data publicly available. CC's work is based upon research supported by the South African Research Chairs Initiative (SARChI) of the Department of Science and Technology (DST), the Square Kilometer Array South Africa (SKA SA) and the National Research Foundation (NRF). The research of BF, KH, DL \& TR have been supported by SARChI, SKA SA and National Astrophysics and Space Science Programme (NASSP) bursaries.

Our findings for NGC 3109 are not an isolated case. Recently, Sánchez-Salcedo, Hidalgo-Gamez \& Martinez-Garcia (2013) studied a sample of slowly rotating gasrich galaxies in the MOND framework. These are again galaxies in the full low acceleration MOND regime. They found at least five such systems (especially NGC 4861 and Holmberg II) that deviate strongly from the MOND predictions, unless their inclinations and distances differ strongly from the nominal values. In the case of NGC 3109, those two parameters are much more constrained which makes it harder to reconcile MOND with the observed kinematics.

Those observations obtained with KAT-7 have shown that despite its relatively small collecting area ( $7 \times 12 \mathrm{~m}$ antennae), this telescope really has a niche for detecting large scale low emission over the $\sim 1^{\circ} \mathrm{FWHM}$ of its antennae. It should be kept in mind that this telescope was built primarily as a 


\section{A. Appendix: Other galaxies in the field}

\section{A.1. ESO 499-G037 (UGCA 196)}

ESO 499-G037 is a SAB(s)d spiral (de Vaucouleurs et al. 1991). It has very bright Hil regions and star formation activity, as seen in the GALEX image (de Paz et al. 2007). Fig. 16 shows the Hi intensity map, superposed on an optical image. We see that the Hi disk has a diameter of $\sim 12.5^{\prime}$, nearly 4 times the optical size (de Vaucouleurs et al. 1991). Because of the low spatial resolution of the KAT-7 data, these observations do not allow to derive a proper RC.

Fig. 17 shows the global profile of ESO 499-G037. From it, a systemic velocity of $953 \pm 3 \mathrm{~km} \mathrm{~s}^{-1}$ is found, similar to the HIPASS determination of $954 \pm 5 \mathrm{~km} \mathrm{~s}^{-1}$ (Koribalski et al. 2004) and the value of 955 $\pm 1 \mathrm{~km} \mathrm{~s}^{-1}$ determined by Barnes \& de Blok (2001). Velocity widths of $\Delta \mathrm{V}_{50}=184 \pm 4 \mathrm{~km} \mathrm{~s}^{-1}$ and $\Delta \mathrm{V}_{20}$ $=200 \pm 4 \mathrm{~km} \mathrm{~s}^{-1}$ are derived. A total flux of $51.7 \pm 5.2 \mathrm{Jy} \mathrm{km} \mathrm{s}^{-1}$ is measured, which is $\sim 25 \%$ larger than the HIPASS flux of $40.2 \pm 4.2 \mathrm{Jy} \mathrm{km} \mathrm{s}^{-1}$ but consistent with the value of $49 \pm 2 \mathrm{Jy} \mathrm{km} \mathrm{s}{ }^{-1}$ from Barnes \& de Blok (2001), also using the Parkes multi-beam system, or the Green Bank value of $48.4 \pm 2.4$ Jy $\mathrm{km} \mathrm{s}^{-1}$ (Springob et al. 2005).

\section{A.2. ESO 499-G038 \& the HI cloud}

ESO 499-G038 is a late-type Sc galaxy with, as can be seen in Fig. 18, a very extended Hi component and a likely associated $\mathrm{HI}$ cloud to the NW, as shown by the faintest contour at $2.5 \times 10^{18} \mathrm{~cm}^{-2}$. While the velocity field shows clear rotation, it is not possible to say more about the kinematics with the present data. Our spatial resolution is sufficient, however, to nearly resolve the two components in the global Hi profile shown in Fig. 19,

We measured a systemic velocity of $871 \pm 4 \mathrm{~km} \mathrm{~s}^{-1}$ for ESO 499-G038 and $912 \pm 5 \mathrm{~km} \mathrm{~s}^{-1}$ for the HI cloud. These are surely better estimates then the values of $885 \pm 5 \mathrm{~km} \mathrm{~s}^{-1}$ (Koribalski et al. 2004) and 888 $\pm 3 \mathrm{~km} \mathrm{~s}^{-1}$ (Barnes \& de Blok 2001) given for the systemic velocity of ESO 499-G038 from their unresolved multi-beam profiles. Fluxes of $9.4 \pm 0.9$ and $1.5 \pm 0.2 \mathrm{Jy} \mathrm{km} \mathrm{s}^{-1}$ are measured for the galaxy and the cloud. This can be compared to $9.5 \pm 2$ (Koribalski et al. 2004) and $11.4 \pm 1 \mathrm{Jy} \mathrm{km} \mathrm{s}^{-1}$ (Barnes \& de Blok 2001) from the unresolved Parkes' spectra. 


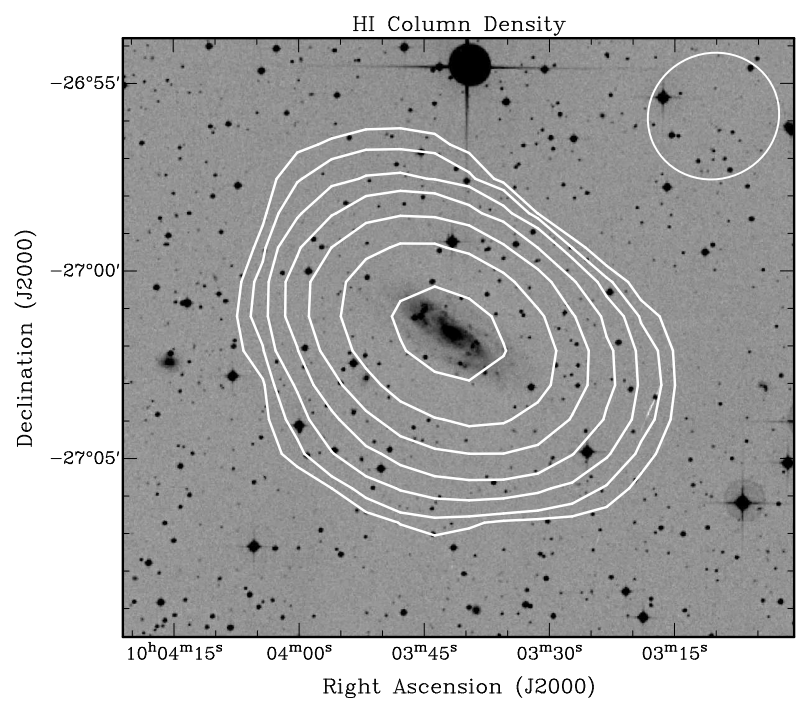

Fig. 16. - KAT-7 data on ESO 499-G037. The Hi emission is superposed on a DSS B image The contours are $0.1,0.2,0.4,0.8,1.6,3.2,6.4 \times 10^{20}$ atoms $\mathrm{cm}^{-2}$.

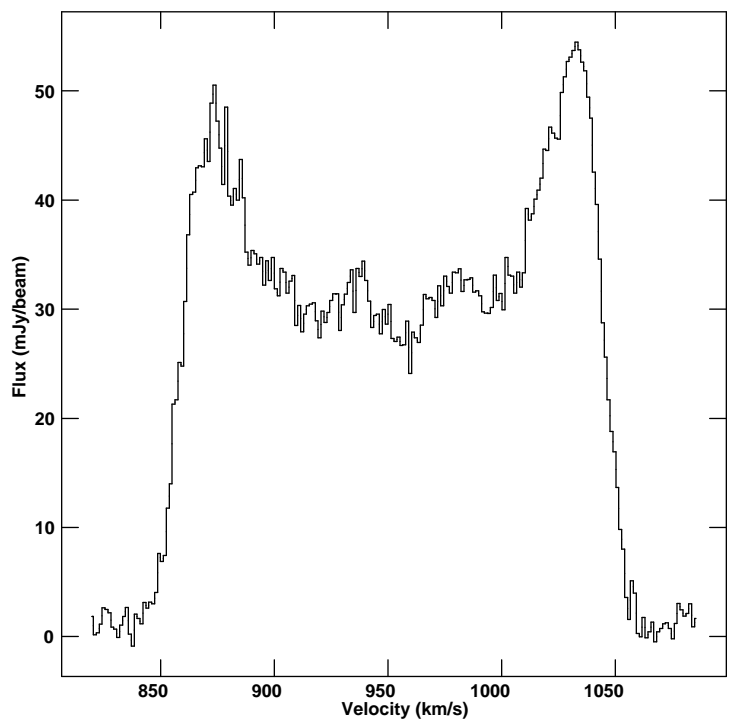

Fig. 17.- Global Hi profile for ESO 499-G037. 


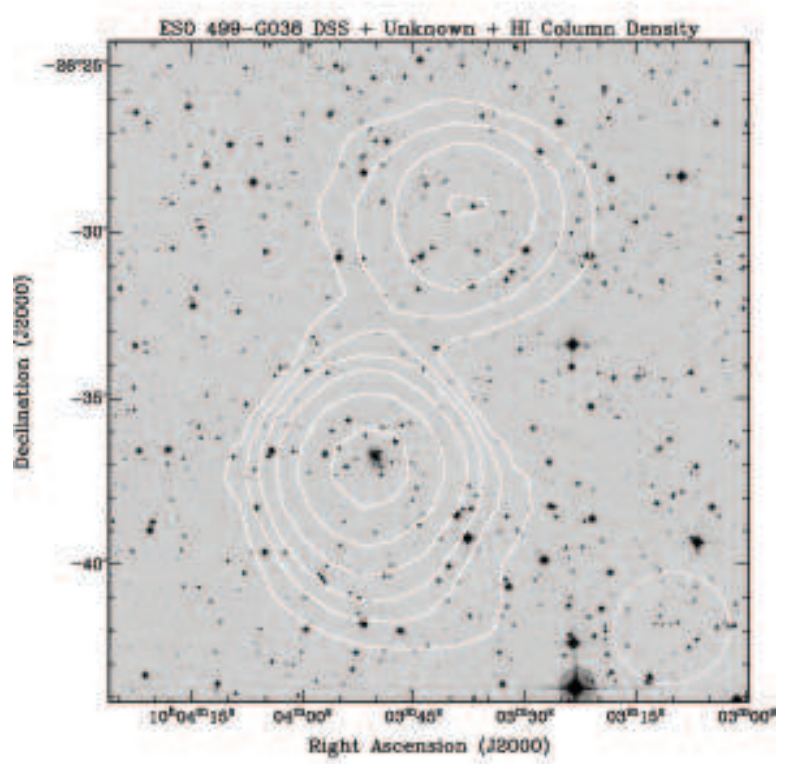

Fig. 18. - KAT-7 data on ESO 499-G038. The Hi emission of the galaxy and the cloud to the NW are superposed on a DSS B image. The contours are $0.025,0.1,0.2,0.4,0.8,1.6 \times 10^{20}$ atoms $\mathrm{cm}^{-2}$.
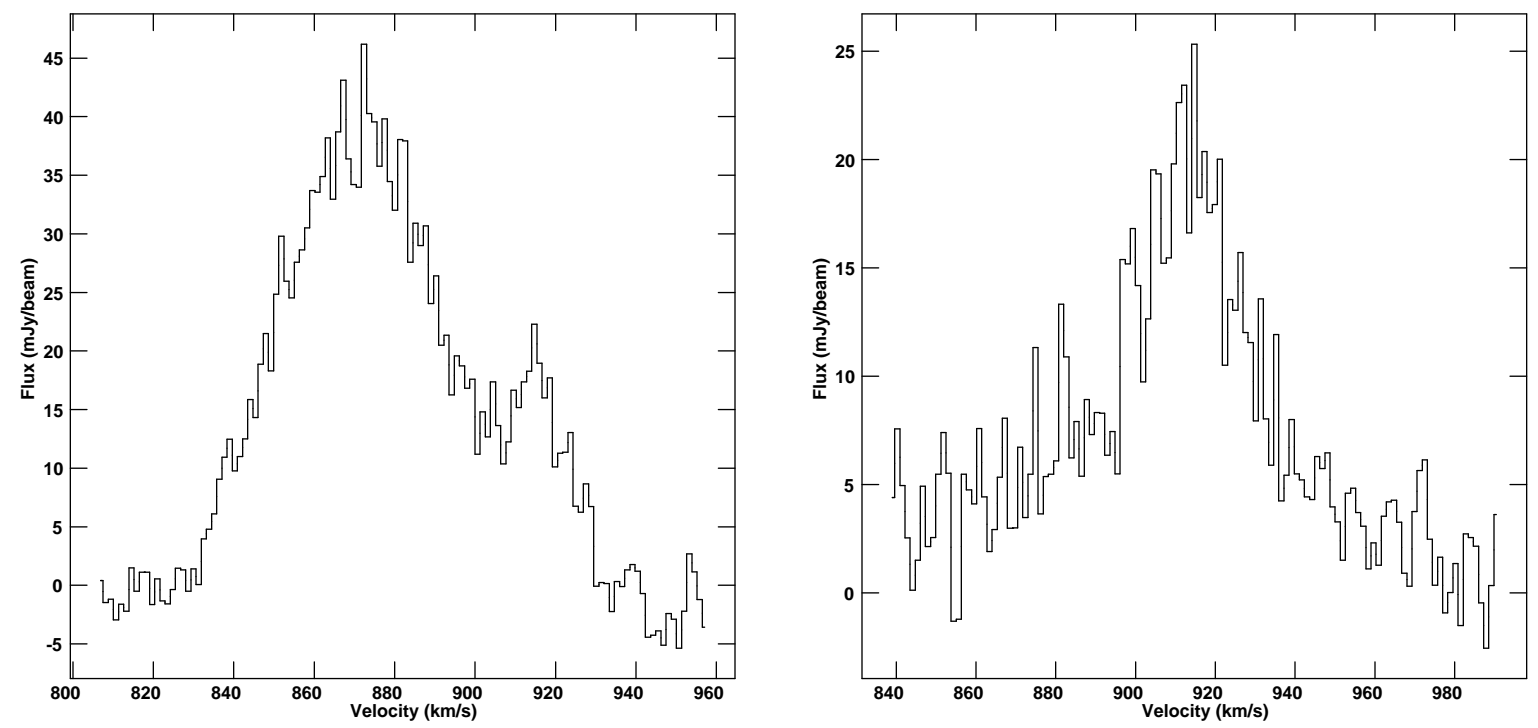

Fig. 19.- Global Hi profile for ESO 499-G038 $\left(\sim 870 \mathrm{~km} \mathrm{~s}^{-1}\right)$ and the cloud to the NW $\left(\sim 910 \mathrm{~km} \mathrm{~s}^{-1}\right)$. 


\section{REFERENCES}

Aparicio, A., Dalcanton, J. J., Gallart, C., \& Martinez-Delgado, D. 1997, AJ, 114, 1447

Barnes, D. G., \& de Blok, W. J. G. 2001, AJ, 122, 825

Begeman, K. G. 1989, A\&A, 223, 47

Begeman, K. G., Broeils, A. H., \& Sanders, R. H. 1991, MNRAS, 249, 523

Bell, E. F., \& de Jong, R. S. 2001, ApJ, 550, 212

Blais-Ouellette, S., Amram, P., \& Carignan, C. 2001, AJ, 121, 1952

Bottema, R., Pestana, J. L. G., Rothberg, B., \& Sanders, R. H. 2002, A\&A, 393, 453

Carignan, C. 1985, AJ, 299, 59

Carignan, C., \& Freeman, K. C. 1985, ApJ, 294, 494

Carignan, C., Freeman, K.C., 1988, ApJ, 332, L33

Carignan, C., Purton, C., 1998, AJ, 506, 125

Capaccioli, M., Piotto, G.,\& Bresolin, F. 1992, AJ, 103,1151

Côté, S., Carignan, C., \& Freeman, K. C. 2000, AJ, 120, 3027

Côté, S., Carignan, C., Sancisi, R., 1991, AJ, 102, 904

Dean, J. F., \& Davies, R. D. 1975, MNRAS, 170, 503

de Blok W.J.G. 2010, AdAst2010E, 5

de Blok W.J.G., McGaugh S.S., \& Rubin V. C. 2001, AJ, 122, 2396

de Blok W. J. G., McGaugh S. S., Bosma A., \& Rubin V. C. 2001, ApJ, 552, L23

de Paz, A. G. et al. 2007, ApJS, 173, 185

de Vaucouleurs, G., de Vaucouleurs, A., Corwin, H. G., Jr, Buta, R. J., Paturel, G., \& Fouqué, P. 1991, Third Reference Catalogue of Bright Galaxies, Vols. 1-3 (Berlin: Springer)

de Villiers, M. 2007, A\&A, 469, 793
Epstein, E. E. 1964, AJ, 69, 490

Famaey, B., \& McGaugh, S. 2013, arXiv1301.0623

Fouqué, P., Bottinelli, L., Durand, N., Gouguenheim, L., \& Paturel, G. 1990, A\&AS, 86, 473

Governato, F. et al. 2010, Nature, 463, 203

Governato, F. et al. 2012, MNRAS, 422, 1231

Grcevich, J., \& Putman, M.E. 2009, ApJ. 696, 385

Grebel, E. K., Gallagher, J. S. III, \& Harbeck, D. 2003, AJ, 125, 1926

Greisen, E. W. 2003, Astrophysics and Space Science Library, 285, 109

Haynes, M. P., Giovanelli, R., \& Roberts, M. S. 1979, ApJ, 229, 83

Hibbard, J.E., van Gorkom, J. H., Rupen, M. P., \& Schiminovich, D. 2001, in Gas and Galaxy Evolution (ASP Conf. Ser. 240) ed. J. E. Hibbard, M. P. Rupen \& J. H. van Gorkom (San Francisco, CA: ASP), 657

Huchtmeier, W. 1973, A\&A, 22, 27

Huchtmeier, W., Seiradakis, J. H., \& Materne, J. 1980, A\&A, 91, 341

Jobin, M., \& Carignan, C. 1990, AJ, 100, 648

King, I. R. 1966, AJ, 71, 64

Koribalski, B. S. et al. 2004, AJ, 128, 16

Kuzio de Naray R., \& Spekkens, K. 2011, ApJ, 741, L29

Kuzio de Naray R., Martinez, G.D., Bullock, J.S., \& Kaplinghat, M. 2010, ApJ, 710, L161

Lake, G. 1989, ApJ, 345, L17

Lake, G., \& Skillman, E. D. 1989, AJ, 98, 1274

Lewis, G. F. et al. 2013, ApJ, 763, 4L

Marchesini D., D'Onghia E., Chincarini G., Firmani C., Conconi P., Molinari E., \& Zacchei A. 2002, ApJ, 575, 801

Mateo, M. 1998, ARA\&A, 36, 435 
McMullin, J. P., Waters, B., Schiebel, D., Young, W., \& Golap, K. 2007, Astronomical Data Analysis Software and Systems XVI, 376, 127

Mihos, J. C., Keating, K.M., Holley-Bockelmann, K., Pisano, D. J., \& Kassim, N. E. 2012, ApJ, 761,186

Milgrom, M. 1983, ApJ, 270, 365

Milgrom, M. 1988, ApJ, 333,689

Musella, I., Piotto, G., \& Capaccioli, M. 1997, AJ, 114,976

Navarro J. F., Frenk C. S., \& White S. D. M. 1996, ApJ, 462, 563

Navarro J. F., Frenk C. S., \& White S. D. M. 1997, ApJ, 490, 493

Ott, J. et al. 2012, AJ, 144, 123

Penny, S. J., Pimbblet, K. A., Conselice, C. J., Brown, M. J. I., Grtzbauch, R., \& Floyd, D. J. E. 2012, ApJ, 758, 32

Pietrzyński, G. et al. 2006, ApJ, 648, 366

Pimbblet, K. A., \& Couch, W. J. 2012, MNRAS, 419,1153

Pontzen, A., \& Governato, F. 2012, MNRAS, 421, 3464

Randriamampandry, T. H. 2013, Msc thesis, University of Cape Town

Sánchez-Salcedo, F. J., Hidalgo-Gámez, A. M., \& Martínez-García, E. E. 2013, AJ, 145, 61

Sancisi, R., Fraternali, F., Oosterloo, T., \& van der Hulst, T. 2008, A\&AR, 15, 189

Sandage, A. 1961, The Hubble Atlas of Galaxies, (Washington: Carnegie Institution), p. 39

Sanders, R. H. 1986, MNRAS, 223, 539

Sanders, R. H. 1996, ApJ, 473, 117

Sault R. J., Teuben P. J., \& Wright M. C. H. 1995. In Astronomical Data Analysis Software and Systems IV, ed. R. Shaw, H.E. Payne, J.J.E. Hayes, ASP Conference Series, 77, 433

Skillman, E. D., Bothun, G. D., Murray, M. A., \& Warmels, R. H. 1987, A\&A, 185, 61
Soszyński, I., Gieren, W., Pietrzyński, G., Bresolin, F., Kudritzki, R.-P., \& Strom, J. 2006, ApJ, 648, 375

Springob, C. M., Haynes, M. P., Giovanelli, R., \& Kent, B. R. 2005, ApJS, 160, 149

van Damme, K. J. 1966, Aust. J. Phys, 19, 687

van der Hulst, J. M., Terlouw, J. P., Begeman, K., Zwitser, W., \& Roelfsema, P.R. 1992, in ASP Conf. Ser., Vol. 25, P. 131

van den Bergh, S. 1994, AJ, 107, 1328

Springel, V., Frenk, C. S., \& White, S. D. M. 2006, Nature, 440, 1137

Whiteoak, J. B., \& Gardner, F. F. 1977, Aust. J. Phys, 30, 187

Yun, M. S., Ho, P. T. P., \& Lo, K. Y. 1994, Nature, 372,530

Zhao, H. S., \& Famaey, B. 2006, ApJ, 638, L9

This 2-column preprint was prepared with the AAS LATEX macros v5.2. 